\title{
COMPLETE CHARACTERIZATION OF OPENNESS, METRIC REGULARITY, AND LIPSCHITZIAN PROPERTIES OF MULTIFUNCTIONS
}

\author{
BORIS MORDUKHOVICH
}

\begin{abstract}
We consider some basic properties of nonsmooth and set-valued mappings (multifunctions) connected with open and inverse mapping principles, distance estimates to the level sets (metric regularity), and a locally Lipschitzian behavior. These properties have many important applications to various problems in nonlinear analysis, optimization, control theory, etc., especially for studying sensitivity and stability questions with respect to perturbations of initial data and parameters. We establish interrelations between these properties and prove effective criteria for their fulfillment stated in terms of robust generalized derivatives for multifunctions and nonsmooth mappings. The results obtained provide complete characterizations of the properties under consideration in a general setting of closed-graph multifunctions in finite dimensions. They ensure new information even in the classical cases of smooth single-valued mappings as well as multifunctions with convex graphs.
\end{abstract}

\section{INTRODUCTION}

Let $\Phi: \mathbf{R}^{n} \rightarrow \mathbf{R}^{m}$ be a finite dimensional mapping strictly differentiable at the point $\bar{x}$ with the derivative (Jacobian) $\nabla \Phi(\bar{x}) \in \mathbf{R}^{m \times n}$, i.e.,

$$
\lim _{x, x^{\prime} \rightarrow \bar{x}}\left\|x-x^{\prime}\right\|^{-1}\left[\Phi(x)-\Phi\left(x^{\prime}\right)-\nabla \Phi(\bar{x})\left(x-x^{\prime}\right)\right]=0 .
$$

One of the key results of the classical differential calculus is the so-called open mapping theorem (principle). It means that

$$
\Phi(\bar{x}) \in \text { int } \Phi(U) \text { for any neighborhood } U \text { of } \bar{x}
$$

if the following surjectivity condition is fulfilled:

$$
\nabla \Phi(\bar{x}) \mathbf{R}^{n}=\mathbf{R}^{m} .
$$

This result, valid also in Banach spaces, is sometimes referred as the interior mapping theorem or the Graves theorem; see, for instance, [11, 16-18]. In [46], Pourciau proves that the result (1.1) is fulfilled for locally Lipschitzian functions $\Phi: \mathbf{R}^{n} \rightarrow \mathbf{R}^{m}$ under the condition

$$
\text { rank } A=m \text { for all } A \in J_{C} \Phi(\bar{x}), m \leq n,
$$

Received by the editors July 23, 1991.

1991 Mathematics Subject Classification. Primary 49J52, 58C06, 58C20; Secondary 49K40, 90C48, 46A30.

Key words and phrases. Nonsmooth analysis, multifunctions, open mapping principle, metric regularity, Lipschitzian behavior, optimization.

This research was supported by the National Science Foundation under grant DMS-9006224. 
stated in terms of Clarke's generalized Jacobian $J_{C} \Phi(\bar{x}) \subset \mathbf{R}^{m \times n}$ [8, p. 70].

Note that, already from the proof of the Ljusternik tangent space theorem [34], one can distill the following fact: if $\Phi$ is strictly differentiable at $\bar{x}$ and the surjectivity condition (1.2) is fulfilled, then there exist a constant $a>0$ and a neighborhood $U$ of $\bar{x}$ such that

$$
B_{a r}(\Phi(x)) \subset \Phi\left(B_{r}(x)\right) \text { for any } B_{r}(x) \subset U,
$$

where $B_{\rho}(z)$ means the closed ball with center $z$ and radius $\rho$.

The property (1.4) is stronger than (1.1) due to two points: (i) the inclusion in (1.4) is uniform with respect to all $x$ in the neighborhood $U$; and (ii) one keeps a linear rate of openness in (1.4) in comparison with "arbitrary openness" in (1.1).

This property (1.4), named covering in a neighborhood, has been studied by Dmitruk, Miljutin, and Osmolovskii [11] for nonsmooth mappings $\Phi: \mathbf{R}^{n} \rightarrow$ $\mathbf{R}^{m}$ which are locally Lipschitzian around $\bar{x}$. It has been proved by Miljutin in $[11, \S 5]$ that the rank condition (1.3) is sufficient for the covering property (1.4), but this condition is far removed from the necessity for $\Phi$ to enjoy (1.4). Some close and more general results for the so-called local surjection property, related to (1.4), have been obtained by Ioffe who has first introduced and evaluated lower estimates for surjection constants (bounds) in various situations (see, e.g., [21 and 23]). In [63], Warga studies a version of (1.4) at the point $\bar{x}$ and proves the refined sufficient conditions for the fulfillment of this property in terms of his "unbounded derivate containers".

Let $\Phi$ be a multifunction (set-valued mapping) from $\mathbf{R}^{n}$ into $\mathbf{R}^{m}$ with the graph

$$
\operatorname{gph} \Phi:=\left\{(x, y) \in \mathbf{R}^{n} \times \mathbf{R}^{m}: y \in \Phi(x)\right\} .
$$

A set-valued counterpart of the covering property (1.4) has been studied by Mordukhovich in [38] and $[39, \S 5]$ where a necessary and sufficient condition for the covering of multifunctions was first obtained. The criterion in [38, Theorem 8] and [39, Corollary 5.2.1] states that if $\Phi$ is an arbitrary multifunction with the closed graph and uniformly bounded values around $\bar{x}$, then the condition

$$
\inf \left\{\left\|x^{*}\right\|: x^{*} \in D^{*} \Phi(\bar{x}, \bar{y})\left(y^{*}\right),\left\|y^{*}\right\|=1, \bar{y} \in \Phi(\bar{x})\right\}>0
$$

is necessary and sufficient for $\Phi$ enjoying the covering property (1.4).

Here $y^{*} \rightarrow D^{*} \Phi(\bar{x}, \bar{y})\left(y^{*}\right)$ is the coderivative of $\Phi$ at $(\bar{x}, \bar{y})$ introduced in Mordukhovich [37] (see $\S 2$ ). If $\Phi$ is single-valued and strictly differentiable at $\bar{x}$, then criterion (1.5) coincides with the classical surjectivity condition (1.2) which appears to be necessary and sufficient for the covering of smooth mappings. If $\Phi: \mathbf{R}^{n} \rightarrow \mathbf{R}^{m}$ is locally Lipschitzian around $\bar{x}$, then criterion (1.5) can be expressed in terms of the subdifferential of the real-valued functions $x \rightarrow\left\langle y^{*}, \Phi(x)\right\rangle$ which was first introduced in [36]; see $\S 2$ for more details.

In [29], Kruger considers a local version of the covering property (1.4) in a neighborhood of a point $(\bar{x}, \bar{y}) \in \operatorname{gph} \Phi$. He proves some characterizations of this property in Banach spaces in terms of the corresponding modifications of the coderivative $D^{*} \Phi(\bar{x}, \bar{y})$. Such a property of multifunctions, called openness at a linear rate around $(\bar{x}, \bar{y})$, has been studied in Borwein and Zhuang [5]. In that paper, the authors provided some criteria for openness in terms of Frankowska's variations of multifunctions [15]. We refer to [4, 16, 23, 39] for 
more information about openness (covering) properties of multifunctions, their modifications, and applications.

Another significant property was actually discovered by Ljusternik [34] and Graves [17] for smooth mappings $\Phi$ with the surjectivity condition (1.2). This is the so-called distance estimate

$$
\operatorname{dist}\left(x, \Phi^{-1}(y)\right) \leq c \operatorname{dist}(y, \Phi(x))
$$

valid for all $x$ from a neighborhood of $\bar{x}$ and for all $y$ from a neighborhood of $\Phi(\bar{x})$, with some constant $c>0$. Here

$$
\operatorname{dist}(x, \Omega):=\inf \{\|x-\omega\|: \omega \in \Omega\}
$$

is the distance function to the set $\Omega$, and

$$
\Phi^{-1}(y):=\left\{x \in \mathbf{R}^{n}: y \in \Phi(x)\right\}
$$

is the inverse mapping to $\Phi$.

It is well known that property (1.6) and its counterparts for set-valued mappings $\Phi$ play a fundamental role in various areas of nonlinear and nonsmooth analysis, optimization, and their applications (see, e.g., $[2-6,10,11,20,25,26$, $39,45-49,58]$ and references therein). In particular, such properties are very important for studying perturbed optimization problems where they provide some stability results and are related to the regularity of constraint systems (the so-called constraint qualifications). The properties of single-valued and multivalued mappings connected with distance estimates like (1.6) are often called the metric regularity of these mappings around the points under consideration. One of the principal results for multifunctions with convex and closed graphs is the Robinson-Ursescu theorem [47,62] on the metric regularity of such multifunctions under an interiority condition.

It has been observed in Dmitruk, Miljutin, and Osmolovskii [11] and in Ioffe [21] that the distance estimate (1.6) for single-valued mappings is equivalent to the covering property (1.4) with $c=1 / a$. Some generalizations of this result for the case of multifunctions have been recently obtained by Borwein and Zhuang [5] and Penot [45] who establish the equivalence between openness with a linear rate around $(\bar{x}, \bar{y}) \in \operatorname{gph} \Phi$ and the metric regularity of $\Phi$ around the same point. Moreover, they prove that these properties of $\Phi$ are equivalent to the so-called pseudo-Lipschitzian property of the inverse mapping $\Phi^{-1}$ around $(\bar{y}, \bar{x})$.

The latter property of multifunctions was introduced by Aubin [1] in connection with inverse mapping theorems and sensitivity analysis of optimization problems. Various applications of this property to significant questions in nonlinear analysis, optimization, and optimal control can be found in Aubin and Ekeland [2], Aubin and Frankowska [3], Dontchev and Hager [12], Mordukhovich [40-43], Rockafellar [54, 56], and elsewhere. This topic is of a great importance for studying stability and sensitivity properties of constraint systems depending on parameters (in particular, for the sets of all feasible solutions or all optimal solutions to a parametrized optimization problem; see, e.g., Fiacco [14]).

The following sufficient condition for pseudo-Lipschitzness of a closed-graph multifunction $\Phi$ around $(\bar{x}, \bar{y}) \in \operatorname{gph} \Phi$ has been obtained by Aubin [1] and Rockafellar [54]:

$$
\left[\left(x^{*}, 0\right) \in N_{C}((\bar{x}, \bar{y}) \mid \operatorname{gph} \Phi)\right] \Rightarrow x^{*}=0
$$


in terms of Clarke's normal cone $[7 ; 8, \mathrm{p} .51]$ to $\operatorname{gph} \Phi$ at $(\bar{x}, \bar{y})$. It follows from the results in Rockafellar [55] that Clarke's cone $N_{C}((\bar{x}, \bar{y}) \mid \operatorname{gph} \Phi)$ is always a (linear) subspace in $\mathbf{R}^{n} \times \mathbf{R}^{m}$ if gph $\Phi$ is a Lipschitzian manifold in the sense of [55], i.e., it is locally representable as the graph of a single-valued Lipschitz continuous function. The class of such multifunctions $\boldsymbol{\Phi}$ covers, besides single-valued locally Lipschitzian functions, all maximal monotone relations (in the sense of Minty [35]) and includes subdifferential mappings for convex functions, saddle functions, strongly subsmooth functions, etc.; see the detailed analysis in [55].

From here one can conclude that relationship (1.7) is never fulfilled for such multifunctions $\Phi$, except the situation when $\operatorname{gph} \Phi$ is locally representable around $(\bar{x}, \bar{y})$ as the graph of a smooth (i.e., strictly differentiable) single-valued function. So, the sufficient condition (1.7) for $\Phi$ being pseudo-Lipschitzian around $(\bar{x}, \bar{y})$ is far removed from the necessity: it does not cover even the case when (nonsmooth) $\Phi$ is single-valued and locally Lipschitzian around $\bar{x}$, i.e., it is pseudo-Lipschitzian automatically.

A necessary and sufficient condition for an arbitrary closed-graph multifunction $\Phi$ to be pseudo-Lipschitzian around $(\bar{x}, \bar{y}) \in \operatorname{gph} \Phi$ has been obtained by Mordukhovich [40] in the form

$$
D^{*} \Phi(\bar{x}, \bar{y})(0)=\{0\}
$$

involved the coderivative of $\Phi$ at $(\bar{x}, \bar{y})$. The difference between conditions (1.7) and (1.8) is the following: instead of Clarke's normal cone as in (1.7), we use in (1.8) the nonconvex normal cone $N(\cdot \mid \mathrm{gph} \Phi)$ in the sense of [36] whose convex closure coincides with $N_{C}(\cdot \mid \operatorname{gph} \Phi)$. Despite their nonconvexity, the normal cone $N(\cdot \mid \operatorname{gph} \Phi)$ and the coderivative $D^{*} \Phi$ generated by it enjoy some nice properties important for applications (in particular, robustness and a rich calculus; see $\S 2$ for more details). Note that the mentioned operation of taking a convexity hull may worsen these properties considerably.

This paper is devoted to a thorough study of openness (covering), metric regularity, and Lipschitzian properties of multifunctions. We shall consider a variety of openness and regularity concepts for arbitrary multifunctions, both local and nonlocal with respect to their images and domains. Then we shall study interrelations between these properties and their interconnections with a corresponding Lipschitzian behavior of the inverse mappings. One of the objects of this paper is to establish the equivalence between suitable openness, regularity, and Lipschitzian properties of multifunctions in a general setting. The main goal is to provide complete and effective characterizations of these properties in convenient and verifiable terms.

For these purposes we use the above mentioned concept of coderivative for multifunctions [37] and develop the results in Mordukhovich [38-40]. Using this technique, we prove new criteria for each of the properties under consideration and obtain precise formulas (equalities instead of estimates) for evaluating the exact bounds of the corresponding regularity, openness, and Lipschitzian moduli. The results obtained have many significant applications to the stability and sensitivity analysis in optimization and optimal control problems, necessary optimality and controllability conditions in such problems, variational inequalities and generalized equations, general aspects of nonsmooth and nonlinear analysis, etc. Some of these applications can be found in [40-43]. 
The organization of the paper is as follows. In $\S 2$ we define general constructions of the normal cone to a set, the coderivative of a multifunction, and the subdifferential of an extended-real-valued function which provide the tools for obtaining our main theorems. We review some properties of these objects and formulate several important results used broadly in the paper.

Section 3 is devoted to covering and openness properties of multifunctions. We consider three notions of this kind, study their interrelations, and prove necessary and sufficient conditions for the fulfillment of these properties in a general setting. We also provide explicit formulas for evaluating the exact bounds of covering and openness moduli. The results obtained ensure open mapping principles for arbitrary closed-graph multifunctions under the conditions which appear to be precise analogues (equivalences) of the classical surjectivity condition (1.2) in nonsmooth and multivalued settings.

Section 4 deals with the concept of metric regularity for multifunctions. There we introduce several definitions of metric regularity which may be local and nonlocal with respect to the image or domain. We prove that one part of these definitions (local with respect to the domain) is equivalent to the corresponding notions of openness in $\S 3$. Another part of the regularity properties introduced (local with respect to the image) appears to be equivalent to the Lipschitzian properties of the inverse mappings studied in the concluding $\S 5$. In $\S \S 4$ and 5 we obtain comprehensive criteria for the metric regularity and Lipschitzian behavior of multifunctions with evaluating the exact bounds of the corresponding moduli. The results obtained provide necessary and sufficient conditions for an arbitrary closed-graph multifunction possessing the Lipschitzian inverse. For convex-graph multifunction these criteria are equivalent to the interiority condition in the Robinson-Ursescu theorem.

We confine our treatment to the finite dimensional case for two reasons. First of all, we would not like to obscure the basic ideas with technical and notational complications. Furthermore, some of the constructions used and the results obtained (as well as their representations) are peculiar to finite dimensions. To develop this material to Banach space settings one can employ the corresponding infinite dimensional extensions of our derivative-like objects studied in [21, 23, $24,28-32,39,60,61]$ and elsewhere.

Our notation is basically standard. Recall that $\operatorname{cl} \Omega, \operatorname{co} \Omega$, and cone $\Omega:=$ $\{\alpha x: \alpha>0, x \in \Omega\}$ means, respectively, the closure, the convex hull, and the conic hull of the arbitrary nonempty set $\Omega$; the set $B$ is always the unit closed ball of the space in question. For a multifunction $\Phi$ from $\mathbf{R}^{n}$ into $\mathbf{R}^{m}$ we denote its domain, image (range), and kernel by, respectively,

$$
\begin{aligned}
\operatorname{Dom} \Phi: & =\left\{x \in \mathbf{R}^{n}: \Phi(x) \neq \varnothing\right\}, \\
\operatorname{Im} \Phi & :=\{y \in \Phi(x): x \in \operatorname{Dom} \Phi\}, \\
\operatorname{Ker} \Phi: & =\left\{x \in \mathbf{R}^{n}: 0 \in \Phi(x)\right\} .
\end{aligned}
$$

We shall consider the set

$$
\begin{aligned}
\limsup _{x \rightarrow \bar{x}} \Phi(x):=\left\{y \in \mathbf{R}^{m}:\right. & \exists \text { sequences } x_{k} \rightarrow \bar{x}, y_{k} \rightarrow y \\
& \text { with } \left.y_{k} \in \Phi\left(x_{k}\right) \text { for all } k=1,2, \ldots\right\}
\end{aligned}
$$

which is called the Kuratowski-Painlevé upper limit of the multifunction $\Phi(x)$ as $x \rightarrow \bar{x}$. 
If $\varphi: \mathbf{R}^{n} \rightarrow \overline{\mathbf{R}}=[-\infty, \infty]$ is an extended-real-valued function, then, as usual,

$\operatorname{dom} \varphi:=\left\{x \in \mathbf{R}^{n}:|\varphi(x)|<\infty\right\}, \quad$ and $\quad$ epi $\varphi:=\left\{(x, \mu) \in \mathbf{R}^{n+1}: \mu \geq \varphi(x)\right\}$.

We shall use the symbol $\varlimsup$ for the upper limit of such (scalar) functions in the classical sense. The symbols $x(\in \Omega) \rightarrow \bar{x}$ and $x \stackrel{\varphi}{\rightarrow} \bar{x}$ mean, respectively, that $x \rightarrow \bar{x}$ with $x \in \Omega$, and $x \rightarrow \bar{x}$ with $\varphi(x) \rightarrow \varphi(\bar{x})$. The adjoint (transposed) matrix to $A$ is denoted by $A^{*}$. Throughout the paper we use the convention that $\inf \varnothing=\infty, \sup \varnothing=-\infty,\|\varnothing\|=\infty$, and $a+\varnothing=\varnothing+b=\varnothing$ for any vectors $a$ and $b$.

\section{GeNERALIZED NORMALS, CODERIVATIVES, AND SUBDifFERENTIALS}

In this section we present some background material in nonsmooth analysis based on the approach in Mordukhovich [36, 37, 39]. Most of this material with the proofs and discussions of the presented results can be found in [39, Chapter 1].

We use a geometric approach to the generalized differentiation of nonsmooth mappings and start with the definition of a normal cone to an arbitrary set.

Let $\Omega$ be a nonempty set in $\mathbf{R}^{n}$ and

$$
P(x, \Omega):=\{\omega \in \operatorname{cl} \Omega:\|x-\omega\|=\operatorname{dist}(x, \Omega)\}
$$

be the projection (the set of best approximations) of $x$ to $\Omega$.

Definition 2.1. The closed (maybe nonconvex) cone

$$
N(\bar{x} \mid \Omega):=\limsup _{x \rightarrow \bar{x}}[\operatorname{cone}(x-P(x, \Omega))]
$$

is called the normal cone to the set $\Omega$ at the point $\bar{x} \in \operatorname{cl} \Omega$.

If $\Omega$ is a convex set, then the normal cone introduced coincides with the normal cone in the sense of convex analysis [50]. In general, the convex closure of (2.1) coincides with the Clarke normal cone:

$$
N_{C}(\bar{x} \mid \Omega)=\operatorname{cl} \operatorname{co} N(\bar{x} \mid \Omega) .
$$

(This is actually the "proximal normal formula" in [8, Proposition 2.5.7].)

In spite of its nonconvexity, the normal cone (2.1) has a variety of useful properties for the analysis and applications to nonsmooth problems (see [39] and references therein). Moreover, the taking of the convex hull as in (2.2) may essentially spoil some of these properties. It happens, in particular, when one considers the Clarke normal cone to graphs of nonsmooth maps and multifunctions, i.e., it always appears in the coderivative constructions below.

For all $x \in \operatorname{cl} \Omega$ let us consider the so-called Fréchet normal cone

$$
N_{F}(x \mid \Omega):=\left\{x^{*} \in \mathbf{R}^{n}: \varlimsup_{x^{\prime}(\in \Omega) \rightarrow x}\left\|x^{\prime}-x\right\|^{-1}\left\langle x^{*}, x^{\prime}-x\right\rangle \leq 0\right\}
$$

which coincides (in finite dimensions) with the polar to the (Bouligand) contingent cone; see, e.g., Aubin and Ekeland [2, Chapter 7]. The following representation was first obtained in Kruger and Mordukhovich [30, 32] (see also [39, Theorem 1.1]). 
Proposition 2.2. For any $\Omega$ and $\bar{x} \in \mathrm{cl} \Omega$ one has

$$
N(\bar{x} \mid \Omega)=\limsup _{x(\in \operatorname{cl} \Omega) \rightarrow \bar{x}} N_{F}(x \mid \Omega) .
$$

Let us now consider the concept of coderivative for an arbitrary multifunction $\Phi$ from $\mathbf{R}^{n}$ into $\mathbf{R}^{m}$ with the nonempty graph.

Definition 2.3. The multifunction $D^{*} \Phi(\bar{x}, \bar{y})$ from $\mathbf{R}^{m}$ into $\mathbf{R}^{n}$ defined by

$$
D^{*} \Phi(\bar{x}, \bar{y})\left(y^{*}\right):=\left\{x^{*} \in \mathbf{R}^{n}:\left(x^{*},-y^{*}\right) \in N((\bar{x}, \bar{y}) \mid \operatorname{gph} \Phi)\right\}
$$

is called the coderivative of $\Phi$ at the point $(\bar{x}, \bar{y}) \in \operatorname{clgph} \Phi$. The symbol $D^{*} \Phi(\bar{x})\left(y^{*}\right)$ is used in (2.4) when $\Phi$ is single-valued at $\bar{x}$ and $\bar{y}=\Phi(\bar{x})$.

If one replaces the normal cone $(2.1)$ in formula (2.4) by the Clarke normal cone (2.2), then the construction defined

$$
D_{C}^{*} \Phi(\bar{x}, \bar{y})\left(y^{*}\right):=\left\{x^{*} \in \mathbf{R}^{n}:\left(x^{*},-y^{*}\right) \in N_{C}((\bar{x}, \bar{y}) \mid \operatorname{gph} \Phi)\right\}
$$

is called the Clarke coderivative of $\Phi$ at $(\bar{x}, \bar{y})$. This multifunction always has convex values and appears to be a dual object to the so-called (Clarke) derivative of $\Phi$ at $(\bar{x}, \bar{y})$ introduced by Aubin in $[1,2]$ through the Clarke tangent cone to $\operatorname{gph} \Phi$. The coderivative (2.4) is nonconvex-valued and, therefore, it cannot be a dual object to any tangentially generated derivative of multifunctions. On the other hand, it follows from Proposition 2.2 that the coderivative (2.4) for any closed-graph $\Phi$ can be represented as the Kuratowski-Painlevé upper limit of the contingent coderivative for $\Phi$ at the points $(x, y)(\in \operatorname{gph} \Phi) \rightarrow(\bar{x}, \bar{y})$, which is the dual construction to Aubin's contingent derivative [1]. Such a representation of (2.4) has been studied in Ioffe [22] under the name of the approximate coderivative of $\Phi$ at $(\bar{x}, \bar{y})$.

It is easy to prove the following assertions.

Proposition 2.4. Let $\Phi: \mathbf{R}^{n} \rightarrow \mathbf{R}^{m}$ be a strictly differentiable function at $\bar{x}$. Then

$$
D^{*} \Phi(\bar{x})\left(y^{*}\right)=\left\{(\nabla \Phi(\bar{x}))^{*} y^{*}\right\} \quad \text { for all } y^{*} \in \mathbf{R}^{m} \text {. }
$$

Proposition 2.5. Let $\Phi$ be a multifunction from $\mathbf{R}^{n}$ into $\mathbf{R}^{m}$ with the convex graph. Then for any $(\bar{x}, \bar{y}) \in \mathrm{cl} \operatorname{gph} \Phi$ the coderivative (2.4) is represented in the form

$$
\begin{aligned}
D^{*} \Phi(\bar{x}, \bar{y})\left(y^{*}\right) & =\left\{x^{*} \in \mathbf{R}^{n}:\left\langle x^{*}, \bar{x}\right\rangle-\left\langle y^{*}, \bar{y}\right\rangle\right. \\
& \left.=\sup \left[\left\langle x^{*}, x\right\rangle-\left\langle y^{*}, y\right\rangle:(x, y) \in \operatorname{gph} \Phi\right]\right\} .
\end{aligned}
$$

Note that under the assumptions of Propositions 2.2 and 2.4 the coderivative (2.4) coincides with the Clarke coderivative (2.5). This is not true in more general cases. Moreover, in common nonsmooth settings one has

$$
\operatorname{co} D^{*} \Phi(\bar{x}, \bar{y})\left(y^{*}\right) \neq D_{C}^{*} \Phi(\bar{x}, \bar{y})\left(y^{*}\right) \text {. }
$$

Indeed, let $\operatorname{gph} \Phi$ be a nonsmooth Lipschitzian manifold around the point $(\bar{x}, \bar{y})$ in the sense of Rockafellar [55] (see $\S 1$ ). One can derive from [55, Theorem 3.5] that in this case $D_{C}^{*} \Phi(\bar{x}, \bar{y})(0)$ is always a linear subspace of a positive dimension. But due to Theorem 5.7 stated below, we have $D^{*} \Phi(\bar{x}, \bar{y})(0)=\{0\}$ for any multifunction $\Phi$ which is pseudo-Lipschitzian around $(\bar{x}, \bar{y})$. So, the coderivatives (2.4) and (2.5) are quite different for significant and sufficiently broad classes of multifunctions. 
One of the principal properties of the coderivative (2.4), which is of a great importance for applications, is its stability (robustness) with respect to perturbations of the initial data. More precisely, the following assertion holds true.

Proposition 2.6. The coderivative (2.4) always enjoys to be robust in the sense that

$$
D^{*} \Phi(\bar{x}, \bar{y})\left(\bar{y}^{*}\right)=\limsup _{(x, y)(\in \operatorname{cl} \operatorname{gph} \Phi) \rightarrow(\bar{x}, \bar{y})} D^{*} \Phi(x, y)\left(y^{*}\right)
$$

for any multifunctions $\Phi$ and points $(\bar{x}, \bar{y}) \in \operatorname{cl} \operatorname{gph} \Phi, \bar{y}^{*} \in \operatorname{Dom} D^{*} \Phi(\bar{x}, \bar{y})$.

Note that such a robustness may be broken for the Clarke coderivative (see an example in Rockafellar [51, p. 22]). This robustness property is always broken for the contingent coderivative of any multifunction $\Phi$ whose graph is a nonsmooth Lipschitzian manifold around $(\bar{x}, \bar{y})$.

Let us now consider an extended-real-valued function $\varphi: \mathbf{R}^{n} \rightarrow \overline{\mathbf{R}}$ and a point $\bar{x} \in \operatorname{dom} \varphi$. We shall associate with $\varphi$ the multifunction $\Phi$ from $\mathbf{R}^{n}$ into $\mathbf{R}$ defined by

$$
\Phi(x)=E_{\varphi}(x):=\{\mu \in \mathbf{R}: \mu \geq \varphi(x)\} \quad \text { with } \operatorname{gph} \Phi=\operatorname{epi} \varphi .
$$

Definition 2.7. (1) The set

$$
\begin{aligned}
\partial^{-} \varphi(\bar{x}) & :=D^{*} E_{\varphi}(\bar{x}, \varphi(\bar{x}))(1) \\
& =\left\{x^{*} \in \mathbf{R}^{n}:\left(x^{*},-1\right) \in N((\bar{x}, \varphi(\bar{x})) \mid \text { epi } \varphi)\right\}
\end{aligned}
$$

is called the (first-order) subdifferential of $\varphi$ at $\bar{x} \in \operatorname{dom} \varphi$.

(2) The set

$$
\begin{aligned}
\partial^{\infty} \varphi(\bar{x}) & :=D^{*} E_{\varphi}(\bar{x}, \varphi(\bar{x}))(0) \\
& =\left\{x^{*} \in \mathbf{R}^{n}:\left(x^{*}, 0\right) \in N((\bar{x}, \varphi(\bar{x})) \mid \text { epi } \varphi)\right\}
\end{aligned}
$$

is called the singular subdifferential of $\varphi$ at $\bar{x}$.

The sets introduced are always closed, but they may be nonconvex in common settings (e.g., for $\varphi(x)=-|x|$ at $\bar{x}=0$ where $\partial^{-} \varphi(0)=\{-1,1\}$ ). For convex functions $\varphi$ the subdifferential (2.6) coincides with the classical subdifferential of convex analysis. When $\varphi$ is locally Lipschitzian around $\bar{x}$, the set $\partial^{-} \varphi(\bar{x})$ is necessarily nonempty and compact. Then this set is reduced to the singleton $\{\nabla \varphi(\bar{x})\}$ if and only if $\varphi$ is strictly differentiable at $\bar{x}$. Note the following connection

$$
\partial_{C} \varphi(\bar{x})=\operatorname{cl} \operatorname{co}\left[\partial^{-} \varphi(\bar{x})+\partial^{\infty} \varphi(\bar{x})\right]
$$

between subdifferential constructions (2.6) and (2.7) and Clarke's generalized gradient $\partial_{C} \varphi(\bar{x})$ for an arbitrary function $\varphi: \mathbf{R}^{n} \rightarrow \overline{\mathbf{R}}$. (This follows immediately from the definitions and formula (2.2).) One can easily see that the normal cone (2.1) to any set $\Omega$ at $\bar{x} \in \Omega$ is expressed in the form

$$
N(\bar{x} \mid \Omega)=\partial^{-} \delta(\bar{x}, \Omega)=\partial^{\infty} \delta(\bar{x}, \Omega)
$$

where $\delta(\cdot, \Omega)$ is the indicator of $\Omega$, i.e., $\delta(x, \Omega):=0$ if $x \in \Omega$, and $\delta(x, \Omega):=\infty$ if $x \notin \Omega$. On the other hand, we have the following representation [39, Proposition 2.7] of the normal cone

$$
N(\bar{x} \mid \Omega)=\operatorname{cone}\left[\partial^{-} \operatorname{dist}(\bar{x}, \Omega)\right] \text { at } \bar{x} \in \operatorname{cl} \Omega
$$


in terms of the subdifferential of the distance function which is Lipschitzian on $\mathbf{R}^{n}$. These formulas allow us to study properties of the normal cone $(2.1)$, the coderivative (2.4), and the subdifferentials (2.6) and (2.7) simultaneously.

The given subdifferential $\partial^{-} \varphi(\bar{x})$ was first introduced in Mordukhovich [36] by the right-hand formula in (2.6). Properties of this construction have been studied in detail in the book [39] and in some previous publications, where a rich calculus and significant applications have been obtained. We refer also to $[9,21,22,28-32,52,53,57]$ for equivalent (in finite dimensions) limiting representations of the subdifferential (2.6) in terms of the so-called Dini subdifferentials, Fréchet subdifferentials, and proximal subgradients.

Note that the subdifferential mapping (2.6) is a robust construction with respect to perturbations of the initial point $\bar{x}$. Moreover, this subdifferential appears to be the best (minimal) among any robust generalized subdifferentials of nonsmooth functions that satisfy certain natural requirements (see some variants of this result in Ioffe [22, Theorem 9] and Mordukhovich [38, Theorem 4] and [39, Theorem 4.9]). One of these required properties is the following stationary principle which is trivially fulfilled for the subdifferential (2.6).

Proposition 2.8. If $\varphi: \mathbf{R}^{n} \rightarrow \overline{\mathbf{R}}$ has a local minimum at $\bar{x} \in \operatorname{dom} \varphi$, then $0 \in \partial^{-} \varphi(\bar{x})$.

The singular subdifferentials have appeared in Kruger and Mordukhovich [30] and Rockafellar [52] in connection with the study of non-Lipschitzian functions. There one can find the proof of the following assertion (see also [39, Theorem 2.1]).

Proposition 2.9. Let $\varphi: \mathbf{R}^{n} \rightarrow \overline{\mathbf{R}}$ be a lower semicontinuous (l.s.c.) function around $\bar{x} \in \operatorname{dom} \varphi$. Then for $\varphi$ being locally Lipschitzian around $\bar{x}$, it is necessary and sufficient that $\partial^{\infty} \varphi(\bar{x})=\{0\}$.

For any extended-real-valued function $\varphi$ on $\mathbf{R}^{n}$, let us consider the subdifferential multifunction $\partial^{-} \varphi$ from $\mathbf{R}^{n}$ into $\mathbf{R}^{n}$ defined in (2.6) for $x \in \operatorname{dom} \varphi$ and $\partial^{-} \varphi(x):=\varnothing$ for $x \notin \operatorname{dom} \varphi$. Then we can introduce the following construction.

Definition 2.10. The set-valued mapping $\partial^{2,-} \varphi(\bar{x}, \bar{y})$ from $\mathbf{R}^{n}$ into $\mathbf{R}^{n}$ defined by

$$
\begin{aligned}
\partial^{2,-} \varphi(\bar{x}, \bar{y})(u) & :=\left(D^{*} \partial^{-} \varphi\right)(\bar{x}, \bar{y})(u) \\
& =\left\{v \in \mathbf{R}^{n}:(v,-u) \in N\left((\bar{x}, \bar{y}) \mid \operatorname{gph} \partial^{-} \varphi\right)\right\}
\end{aligned}
$$

is called the second-order subdifferential of $\varphi$ at $\bar{x} \in \operatorname{dom} \varphi$ relative to $\bar{y} \in$ $\partial^{-} \varphi(\bar{x})$.

Remark 2.11. For any function $\varphi: \mathbf{R}^{n} \rightarrow \overline{\mathbf{R}}$, we can symmetrically define the (first-order) superdifferential

$$
\partial^{+} \varphi(\bar{x}):=-\partial^{-}(-\varphi)(\bar{x})
$$

of $\varphi$ at $\bar{x} \in \operatorname{dom} \varphi$ and the second-order superdifferential

$$
\partial^{2,+} \varphi(\bar{x}, \bar{y})(u):=-\left(D^{*} \partial^{+} \varphi\right)(\bar{x}, \bar{y})(-u)
$$

of $\varphi$ at $\bar{x}$ relative to $\bar{y} \in \partial^{+} \varphi(\bar{x})$. These superdifferential constructions may be distinguished considerably from the subdifferential ones because differential 
properties of nonsmooth functions $\varphi$ and $-\varphi$ are essentially distinct (see examples in [39, §2]). So, the subdifferentials and superdifferentials introduced are constructions of unilateral analysis (in the sense of Moreau [44]), even in the case of convex (concave) functions. They are able to provide a more precise study of the local behavior of nonsmooth functions than bilateral constructions with the classical symmetry $\partial(-\varphi)(\bar{x})=-\partial \varphi(\bar{x})$ (such as Clarke's generalized gradient for locally Lipschitzian functions or Warga's derivate containers). One can find the results in this vein in Mordukhovich [39]. For the purposes of this paper, it is sufficient to use the subdifferential constructions only.

Note that if $\varphi \in C^{2}$ around $\bar{x}$ with the gradient $\bar{y}=\nabla \varphi(\bar{x})$ and the Hessian matrix $\nabla^{2} \varphi(\bar{x})$, then

$$
\partial^{2,-} \varphi(\bar{x})(u)=\partial^{2,+} \varphi(\bar{x})(u)=\left\{\left(\nabla^{2} \varphi(\bar{x})\right)^{*} u\right\} \quad \text { for any } u \in \mathbf{R}^{n} .
$$

One can also define the Clarke counterpart of the second-order subdifferential (2.9) in the form

$$
\begin{aligned}
\partial_{C}^{2} \varphi(\bar{x}, \bar{y})(u) & :=\left(D_{C}^{*} \partial_{C} \varphi\right)(\bar{x}, \bar{y})(u) \\
& =\left\{v \in \mathbf{R}^{n}:(v,-u) \in N_{C}\left((\bar{x}, \bar{y}) \mid \operatorname{gph} \partial_{C} \varphi\right)\right\}
\end{aligned}
$$

(cf. Aubin [1]). As it follows from the results of Rockafellar [55], the set (2.11) is an affine manifold in $\mathbf{R}^{n}$ for a broad class of functions $\varphi$ including convex, saddle, strongly subsmooth ones, etc.; see $\S 1$. Therefore, in such cases the construction (2.11) cannot actually reflect anything other than classical-like "two-sided" aspects of differentiation. This construction carries, by contrast with (2.9) and (2.10), little information about the local behavior of $\varphi \notin \mathbf{C}^{2}$.

In this paper we use the coderivative (2.4) as the primal tool for the analysis of multifunctions and nonsmooth mappings. For the case of scalar functions $\varphi: \mathbf{R}^{n} \rightarrow \overline{\mathbf{R}}$ this coderivative generates the constructions of the first- and secondorder subdifferentials (2.6), (2.7), and (2.9). On the other hand, if $\Phi: \mathbf{R}^{n} \rightarrow \mathbf{R}^{m}$ is a Lipschitzian vector function, then the coderivative (2.4) can be expressed through the subdifferentials (2.6) of the scalar functions

$$
x \rightarrow\left\langle y^{*}, \Phi\right\rangle(x):=\left\langle y^{*}, \Phi(x)\right\rangle
$$

associated with $\Phi$ for all $y^{*} \in \mathbf{R}^{n}$. The proof of the following result can be found in [22, Proposition 8] and [39, Theorem 3.3].

Proposition 2.12. If $\Phi$ is single-valued and locally Lipschitzian around $\bar{x}$, then one has

$$
D^{*} \Phi(\bar{x})\left(y^{*}\right)=\partial^{-}\left\langle y^{*}, \Phi\right\rangle(\bar{x}) \neq \varnothing \quad \text { for all } y^{*} \in \mathbf{R}^{m} .
$$

Note that the analogue of representation (2.12), stated in terms of Clarke's coderivative and generalized gradient, is never fulfilled for nonsmooth Lipschitzian functions $\Phi$ because in this case $D_{C}^{*} \Phi(\bar{x})(0) \neq\{0\}$. At the same time, we can obtain the interrelation

$$
\operatorname{co} D^{*} \Phi(\bar{x})\left(y^{*}\right)=\left\{A^{*} y^{*}: A \in J_{C} \Phi(\bar{x})\right\} \quad \text { for all } y^{*} \in \mathbf{R}^{m}
$$

between Clarke's generalized Jacobian and the convex hull of the coderivative (2.4) for locally Lipschitzian functions. (The latter formula follows from (2.8), (2.12), and the chain rule in Clarke [8, Theorem 2.6.6].)

Let us now consider some calculus rules for the coderivatives and subdifferentials introduced, which are used in proving the main results of this paper. 
The following theorem is the cornerstone of the generalized differential calculus for multifunctions and nonsmooth mappings.

Theorem 2.13. Let $\Phi_{1}$ and $\Phi_{2}$ be multifunctions from $\mathbf{R}^{n}$ into $\mathbf{R}^{m}$ with the closed graphs, and let $\bar{y} \in \Phi_{1}(\bar{x})+\Phi_{2}(\bar{x})$. Let us assume that the sets

$$
S(x, y):=\left\{\left(y_{1}, y_{2}\right) \in \mathbf{R}^{2 m}: y_{1} \in \Phi_{1}(x), y_{2} \in \Phi_{2}(x), y_{1}+y_{2}=y\right\}
$$

are bounded uniformly around $(\bar{x}, \bar{y})$, and that the following condition is fulfilled :

$$
D^{*} \Phi_{1}\left(\bar{x}, y_{1}\right)(0) \cap\left[-D^{*} \Phi_{2}\left(\bar{x}, y_{2}\right)(0)\right]=\{0\} \quad \forall\left(y_{1}, y_{2}\right) \in S(\bar{x}, \bar{y})
$$

Then

$$
D^{*}\left(\Phi_{1}+\Phi_{2}\right)(\bar{x}, \bar{y})\left(y^{*}\right) \subset \bigcup_{\left(y_{1}, y_{2}\right) \in S(\bar{x}, \bar{y})}\left[D^{*} \Phi_{1}\left(\bar{x}, y_{1}\right)\left(y^{*}\right)+D^{*} \Phi_{2}\left(\bar{x}, y_{2}\right)\left(y^{*}\right)\right]
$$

The theorem formulated is proved in Mordukhovich [41, 43] by using the so-called metric approximation method (abbr. MAM). This method provides special approximations of questions under consideration by parametric families of smooth optimization problems without constraints (see [39, Chapters 1-3] and references therein for more details and various applications). Note that the first introduction of the normal cone (2.1) in [36] (as well as the subdifferential (2.6) and the coderivative (2.5) generated by it) was actually a by-product of using the MAM for obtaining necessary optimality conditions in nonsmooth problems.

The usage of the MAM in the setting of Theorem 2.13 reflects a variational approach to the calculus of coderivatives and subdifferentials developed in [3843]. The following corollary of the theorem was first obtained in [38, Theorem 2]. We refer also to [9, 22, 24, 28, 29, 39, 53, 57] for some related results and discussions.

Corollary 2.14. Let $\varphi_{1}$ and $\varphi_{2}$ be extended-real-valued functions l.s.c. around $\bar{x} \in \operatorname{dom} \varphi_{1} \cap \operatorname{dom} \varphi_{2}$, and let the following condition be fulfilled:

$$
\partial^{\infty} \varphi_{1}(\bar{x}) \cap\left(-\partial^{\infty} \varphi_{2}(\bar{x})\right)=\{0\}
$$

Then one has

$$
\partial^{-}\left(\varphi_{1}+\varphi_{2}\right)(\bar{x}) \subset \partial^{-} \varphi_{1}(\bar{x})+\partial^{-} \varphi_{2}(\bar{x})
$$

Remark 2.15. Due to Proposition 2.9, the condition (2.15) is automatically fulfilled if either $\varphi_{1}$ or $\varphi_{2}$ is locally Lipschitzian around $\bar{x}$. Similarly, a Lipschitzian behavior of one of the multifunctions $\Phi_{1}$ and $\Phi_{2}$ ensures the fulfillment of assumption (2.14) in the general setting of Theorem 2.13 (see Corollary 5.10).

It is easy to obtain a number of other corollaries of Theorem 2.13 considering the multifunctions $\Phi_{1}$ and $\Phi_{2}$ of the special form. In particular, the theorem implies some calculus rules for the singular subdifferentials (2.7), the secondorder semidifferentials (2.9) and (2.10), etc. Now we formulate a result about the subdifferentiation of the marginal function

$$
m(x):=\inf \{\varphi(y): y \in \Phi(x)\}
$$

at $\bar{x} \in \operatorname{dom} m$ important in what follows. For all $x \in \operatorname{dom} m$ we consider the set

$$
M(x):=\{y \in \Phi(x): \varphi(y)=m(x)\}
$$


associated with (2.16) where $\Phi$ is a multifunction from $\mathbf{R}^{n}$ into $\mathbf{R}^{m}$ and $\varphi$ is an extended-real-valued function.

Theorem 2.16. Let the multifunction $\Phi$ have the closed graph, let the sets $M(x)$ be nonempty and uniformly bounded around $\bar{x}$, and let the function $\varphi$ be l.s.c. around any point $\bar{y} \in \Phi(\bar{x})$. If

$$
\partial^{\infty} \varphi(\bar{y}) \cap \operatorname{Ker} D^{*} \Phi(\bar{x}, \bar{y})=\{0\} \quad \text { for any } \bar{y} \in M(\bar{x}),
$$

then one has

$$
\partial^{-} m(\bar{x}) \subset \bigcup_{y^{*}, \bar{y}}\left[D^{*} \Phi(\bar{x}, \bar{y})\left(y^{*}\right): y^{*} \in \partial^{-} \varphi(\bar{y}), \bar{y} \in M(\bar{x})\right] .
$$

The given theorem was obtained in Mordukhovich [38, Theorem 7] and [39, Theorem 4.6] by using the MAM. One can regard this result as a generalized chain rule for the composition of a real function and a multifunction. If, in particular, $\Phi$ is single-valued and locally Lipschitzian around $\bar{x}$, then we have the inclusion

$$
\partial^{-}(\varphi \circ \Phi)(\bar{x}) \subset \bigcup_{y^{*} \in \partial^{-} \varphi(\bar{y})} \partial^{-}\left\langle y^{*}, \Phi\right\rangle(\bar{x})
$$

which follows from (2.18) and Proposition 2.12. Note that condition (2.17) is fulfilled automatically if either $\varphi$ is locally Lipschitzian around $\bar{y}$ or $\Phi(x) \equiv$ $\mathbf{R}^{m}$. The latter case was considered in Rockafellar [53]. Some recent generalizations and applications of Theorem 2.16 can be found in [40].

Let us now consider a corollary of this theorem for the distance function from a fixed point to a variable set

$$
\rho_{v}(x):=\operatorname{dist}(v, \Phi(x))
$$

which is a special case of (2.16) for $\varphi(y)=\|v-y\|$. Note that in this case the set $M(x)$ in Theorem 2.16 coincides with the projection $P(v, \Phi(x))$ of $v$ to $\Phi(x)$. We say that a multifunction $\Phi$ is locally bounded around $\bar{x}$ if there exists a neighborhood $U$ of $\bar{x}$ such that the set $\Phi(U)$ is bounded.

Corollary 2.17. Let $\Phi$ be a closed-graph multifunction from $\mathbf{R}^{n}$ into $\mathbf{R}^{m}$ which is locally bounded around $\bar{x} \in \operatorname{Dom} \Phi$. Then

$\partial^{-} \rho_{v}(\bar{x}) \subset \bigcup\left[D^{*}(\bar{x}, \bar{y})\left(y^{*}\right):\left\|y^{*}\right\| \leq 1,\left\langle y^{*}, \bar{y}-v\right\rangle=\|\bar{y}-v\|, \bar{y} \in P(v, \Phi(\bar{x}))\right]$ where $y^{*}=(\bar{y}-v) /\|\bar{y}-v\|$ if $v \notin \Phi(\bar{x})$.

To conclude this section, let us mention a useful assertion (see [38, Theorem 5] and [39, Theorem 5.1]) which can be considered as an extended variant of Ekeland's variational principle [13] in finite dimensions.

Proposition 2.18. Let $\varphi: \mathbf{R}^{n} \rightarrow(-\infty, \infty]$ be a l.s.c. proper function which is bounded below. Let $p: \mathbf{R}^{n} \rightarrow[0, \infty)$ be a l.s.c. function satisfying the conditions: $p(x) \rightarrow \infty$ as $\|x\| \rightarrow \infty, p(0)=0$, and $p\left(x_{1}+x_{2}\right) \leq p\left(x_{1}\right)+p\left(x_{2}\right)$ for any $x_{1}$ and $x_{2}$ in $\mathbf{R}^{n}$. Let positive numbers $\varepsilon$ and $\lambda$ be given. Then for any $x_{\varepsilon} \in \mathbf{R}^{n}$ with

$$
\varphi\left(x_{\varepsilon}\right) \leq \inf \left\{\varphi(x): x \in \mathbf{R}^{n}\right\}+\varepsilon
$$

there exists a point $x_{\lambda} \in \mathbf{R}^{n}$ such that $\varphi\left(x_{\lambda}\right) \leq \varphi\left(x_{\varepsilon}\right), p\left(x_{\lambda}-x_{\varepsilon}\right) \leq \lambda$, and

$$
\varphi\left(x_{\lambda}\right) \leq \varphi(x)+(\varepsilon / \lambda) p\left(x-x_{\lambda}\right) \text { for all } x \in \mathbf{R}^{n} \text {. }
$$


The proof of this assertion is quite elementary (cf. Hiriart-Urruty [19] for $p(x)=\|x\|)$ : the desired vector $x_{\lambda}$ is a solution of the unconditional minimization problem:

$$
\varphi(x)+(\varepsilon / \lambda) p\left(x-x_{\varepsilon}\right) \rightarrow \inf , \quad x \in \mathbf{R}^{n},
$$

which does exist due to the classical Weierstrass theorem in finite dimensions.

\section{COVERING AND OPENNESS OF MULTIFUNCTIONS}

In the rest of the paper, $\Phi$ is a multifunction from $\mathbf{R}^{n}$ into $\mathbf{R}^{m}$ with the closed graph, $\bar{x} \in \operatorname{Dom} \Phi$, and $(\bar{x}, \bar{y}) \in \operatorname{gph} \Phi$. In this section we consider three interrelated notions of covering (openness) for multifunctions and obtain their complete characterization. Let us begin with the covering property for $\Phi$ around $\bar{x}$ which is nonlocal with respect to $\Phi(\bar{x})$.

Definition 3.1. We say that $\Phi$ enjoys the covering property around $\bar{x}$ if there exist a number $a>0$ and a neighborhood $U$ of $\bar{x}$ such that for any $(x, r)$ with $B_{r}(x) \subset U$ one has $B_{a r}(\Phi(x)) \subset \Phi\left(B_{r}(x)\right)$. Each of such numbers $a$ (corresponding to different neighborhoods) is called the covering modulus for $\Phi$ around $\bar{x}$. The supremum of all covering moduli is called the covering bound for $\Phi$ around $\bar{x}$ and is denoted by $(\operatorname{cov} \Phi)(\bar{x})$.

Note that $(\operatorname{cov} \Phi)(\bar{x})$ is the least upper bound of all covering moduli for $\Phi$ around $\bar{x}$ but may not be a covering modulus itself. Our goal is to prove effective criteria for the covering property and to evaluate the covering bound in a general setting. Consider the numbers

$$
a(\Phi, \bar{x}):=\inf \left\{\left\|x^{*}\right\|: x^{*} \in D^{*} \Phi(\bar{x}, \bar{y})\left(y^{*}\right),\left\|y^{*}\right\|=1, \bar{y} \in \Phi(\bar{x})\right\},
$$

$$
c(\Phi, \bar{x}):=\inf \left\{c:\left\|y^{*}\right\| \leq c\left\|x^{*}\right\| \text { if } x^{*} \in D^{*} \Phi(\bar{x}, \bar{y})\left(y^{*}\right) \text { and } \bar{y} \in \Phi(\bar{x})\right\} .
$$

Proposition 3.2. If $a(\Phi, \bar{x})>0$, then $c(\Phi, \bar{x})=1 / a(\Phi, \bar{x})$.

Proof. First let us consider the extreme case $a(\Phi, \bar{x})=\infty$. This means that $D^{*} \Phi(\bar{x}, \bar{y})\left(y^{*}\right)=\varnothing$ if $y^{*} \neq 0$. Therefore, the implication

$$
\left[x^{*} \in D^{*} \Phi(\bar{x}, \bar{y})\left(y^{*}\right), \bar{y} \in \Phi(\bar{x})\right] \Rightarrow\left\|y^{*}\right\| \leq c\left\|x^{*}\right\|
$$

holds for $c=0$ only, i.e., $c(\Phi, \bar{x})=0$ in (3.2).

Let $0<a(\Phi, \bar{x})<\infty$. Now we shall prove that implication (3.3) is fulfilled for any number $c>1 / a(\Phi, \bar{x})$. If it is not true, one can find vectors $\bar{y} \in$ $\Phi(\bar{x}), \bar{y}^{*} \in \mathbf{R}^{m}$, and $\bar{x}^{*} \in D^{*} \Phi(\bar{x}, \bar{y})\left(\bar{y}^{*}\right)$ such that $\left\|\bar{y}^{*}\right\|>c\left\|\bar{x}^{*}\right\|$. Setting $y^{*}:=\bar{y}^{*} /\left\|\bar{y}^{*}\right\|$ and $x^{*}:=\bar{x}^{*} /\left\|\bar{y}^{*}\right\|$, we have

$$
\left\|y^{*}\right\|=1, \quad x^{*} \in D^{*} \Phi(\bar{x}, \bar{y})\left(y^{*}\right), \quad \text { and } \quad\left\|x^{*}\right\|<1 / c<a(\Phi, \bar{x})
$$

which contradicts the definition of $a(\Phi, \bar{x})$ in (3.1). Therefore, (3.3) holds for any $c>1 / a(\Phi, \bar{x})$. This implies the inequality $c(\Phi, \bar{x}) \leq 1 / a(\Phi, \bar{x})$.

Let us prove the opposite inequality $c(\Phi, \bar{x}) \geq 1 / a(\Phi, \bar{x})$. Consider a number $c>0$ satisfying (3.3) and vectors $\bar{y} \in \Phi(\bar{x}), x^{*} \in D^{*} \Phi(\bar{x}, \bar{y})\left(y^{*}\right)$ with $\left\|y^{*}\right\|=1$. It follows from (3.3) that $\left\|x^{*}\right\| \geq 1 / c$. Therefore, $c \geq 1 / a(\Phi, \bar{x})$ for any number $c>0$ satisfying (3.3). This implies the inequality $c(\Phi, \bar{x}) \geq$ $1 / a(\Phi, \bar{x})$ and ends the proof of the proposition.

Now we obtain several criteria for the covering property with evaluating the covering bound for $\Phi$ around $\bar{x}$. 
Theorem 3.3. Let $\Phi$ be locally bounded around $\bar{x}$. Then the following conditions are equivalent:

(a) $\Phi$ enjoys the covering property around $\bar{x}$.

(b) $a(\Phi, \bar{x})>0$.

(c) There exists a number $c>0$ and a neighborhood $U$ of $\bar{x}$ such that

$$
\left\|y^{*}\right\| \leq c\left\|x^{*}\right\| \text { for any } x^{*} \in D^{*} \Phi(x, y)\left(y^{*}\right), x \in U \text {, and } y \in \Phi(x) \text {. }
$$

(d) There exists a neighborhood $U$ of $\bar{x}$ such that

$\operatorname{Ker} D^{*} \Phi(x, y)=\{0\} \quad$ for any $x \in U$ and $y \in \Phi(x)$.

(e) $\operatorname{Ker} D^{*} \Phi(\bar{x}, \bar{y})=\{0\}$ for all $\bar{y} \in \Phi(\bar{x})$.

When these properties hold, one has

$$
(\operatorname{cov} \Phi)(\bar{x})=a(\Phi, \bar{x})=1 / c(\Phi, \bar{x})
$$

Proof. Let (a) be fulfilled with a covering modulus $a>0$. We shall show that $a \leq a(\Phi, \bar{x})$ which implies (a) $\Rightarrow$ (b). Proving by contradiction, suppose that $a>a(\Phi, \bar{x})$. Then for any small number $\gamma>0$ we can find vectors $x^{*} \in \mathbf{R}^{n}$, $y^{*} \in \mathbf{R}^{m}$, and $\bar{y} \in \Phi(\bar{x})$ such that

$$
\left(x^{*},-y^{*}\right) \in N((\bar{x}, \bar{y}) \mid \operatorname{gph} \Phi), \quad\left\|y^{*}\right\|=1, \quad \text { and } \quad\left\|x^{*}\right\|<a-\gamma .
$$

Due to Proposition 2.2, there exist sequences $x_{k} \rightarrow \bar{x}, y_{k} \rightarrow \bar{y}, x_{k}^{*} \rightarrow x^{*}$, and $y_{k}^{*} \rightarrow y^{*}$ as $k \rightarrow \infty$ such that

$$
\left(x_{k}^{*},-y_{k}^{*}\right) \in N_{F}\left(\left(x_{k}, y_{k}\right) \mid \operatorname{gph} \Phi\right) \text { and }\left(x_{k}, y_{k}\right) \in \operatorname{gph} \Phi \quad \text { for } k=1,2, \ldots
$$

Without loss of generality, $\left\|y_{k}^{*}\right\|=1$ and $\left\|x_{k}^{*}\right\|<a-\gamma$ for all $k=1,2, \ldots$. Using formula (2.3), we have

$$
\underset{\substack{(x, y) \rightarrow\left(x_{k}, y_{k}\right) \\ y \in \Phi(x)}}{\lim \sup _{k}} \frac{\left\langle x_{k}^{*}, x-x_{k}\right\rangle-\left\langle y_{k}^{*}, y-y_{k}\right\rangle}{\left\|\left(x-x_{k}, y-y_{k}\right)\right\|} \leq 0 .
$$

For convenience let us set $\|(x, y)\|:=\|x\|+\|y\|$. Then formula (3.6) implies that for any $\varepsilon>0$ one can find a sequence $\delta_{k} \downarrow 0$ as $k \rightarrow \infty$ such that

$$
\left\langle y_{k}^{*}, y-y_{k}\right\rangle+\varepsilon\left(\left\|x-x_{k}\right\|+\left\|y-y_{k}\right\|\right) \geq\left(x_{k}^{*}, x-x_{k}\right\rangle \text { for } k=1,2, \ldots
$$

if $(x, y) \in \operatorname{gph} \Phi$ with $\left\|x-x_{k}\right\| \leq \delta_{k}$ and $\left\|y-y_{k}\right\| \leq \delta_{k}, k=1,2, \ldots$.

Let us select the sequence $\left\{\delta_{k}\right\}$ for $\varepsilon=\gamma /(1+a)$ and let us set

$$
r_{k}:=\min \left\{\delta_{k}, \delta_{k} / a\right\} \quad \text { as } k=1,2, \ldots
$$

Considering sequences of vectors

$$
z_{k}:=y_{k}-a r_{k} v_{k} \quad \text { with }\left\|v_{k}\right\|=1, \quad\left\langle y_{k}^{*}, v_{k}\right\rangle=1 \quad \text { for } k=1,2, \ldots,
$$

we have $\left\|z_{k}-y_{k}\right\| \leq a r_{k} \leq \delta_{k}$ and

$$
\begin{aligned}
\left\langle y_{k}^{*}, z_{k}-y_{k}\right\rangle+\varepsilon\left(\left\|x-x_{k}\right\|+\left\|z_{k}-y_{k}\right\|\right) & =-a r_{k}+\frac{\gamma}{1+a}\left(\left\|x-x_{k}\right\|+a r_{k}\right) \\
& \leq-(a-\gamma) r_{k}<\left\langle x_{k}^{*}, x-x_{k}\right\rangle
\end{aligned}
$$

for all $x \in \mathbf{R}^{n}$ such that $\left\|x-x_{k}\right\| \leq r_{k}$ as $k=1,2, \ldots$. Hence it follows that $z_{k} \notin \Phi(x)$ for all $x \in B_{r_{k}}\left(x_{k}\right)$, otherwise it contradicts (3.6). 
So, there exist sequences $\left\{x_{k}\right\},\left\{r_{k}\right\},\left\{y_{k}\right\}$, and $\left\{z_{k}\right\}$ such that $x_{k} \rightarrow \bar{x}$, $r_{k} \downarrow 0$ as $k \rightarrow \infty$, and

$$
y_{k} \in \Phi\left(x_{k}\right), \quad\left\|z_{k}-y_{k}\right\| \leq a r_{k}, \quad z_{k} \notin \Phi(x) \quad \text { for any } x \in B_{r_{k}}\left(x_{k}\right) .
$$

The relations obtained mean that the number $a>0$ cannot be a covering modulus for $\Phi$ in any neighborhood $U$. This contradiction proves the required inequality $a \leq a(\Phi, \bar{x})$ and implication $(\mathrm{a}) \Rightarrow(\mathrm{b})$.

Let us prove $(b) \Rightarrow(c)$. Suppose that (c) does not hold. Then for any sequence of positive numbers $c_{k} \rightarrow \infty$ as $k \rightarrow \infty$ we can find sequences of vectors $\left\{x_{k}\right\},\left\{y_{k}\right\},\left\{x_{k}^{*}\right\}$, and $\left\{y_{k}^{*}\right\}$ such that $x_{k} \rightarrow \bar{x}$ and

$y_{k} \in \Phi\left(x_{k}\right), \quad x_{k}^{*} \in D^{*} \Phi\left(x_{k}, y_{k}\right)\left(y_{k}^{*}\right), \quad\left\|y_{k}^{*}\right\|>c_{k}\left\|x_{k}^{*}\right\| \quad$ for $k=1,2, \ldots$

As $\left\|y_{k}^{*}\right\|>0$, we set $\tilde{y}_{k}^{*}:=y_{k}^{*} /\left\|y_{k}^{*}\right\|$ and $\tilde{x}_{k}^{*}:=x_{k}^{*} /\left\|y_{k}^{*}\right\|$. Then one has

$$
\tilde{x}_{k}^{*} \in D^{*} \Phi\left(x_{k}, y_{k}\right)\left(\tilde{y}_{k}^{*}\right), \quad\left\|\tilde{y}_{k}^{*}\right\|=1, \quad\left\|\tilde{x}_{k}^{*}\right\|<1 / c_{k} \quad \text { for } k=1,2, \ldots
$$

Due to the boundedness of the sequences $\left\{y_{k}\right\},\left\{\tilde{y}_{k}^{*}\right\}$, and $\left\{\tilde{x}_{k}^{*}\right\}$, one can assume that they converge themselves to vectors $\bar{y} \in \Phi(\bar{x}), y^{*} \in \mathbf{R}^{m}$, and $0 \in \mathbf{R}^{n}$ respectively. Passing to the limit as $k \rightarrow \infty$ and using the robustness of the coderivative (Proposition 2.6), we get

$$
0 \in D^{*} \Phi(\bar{x}, \bar{y})\left(y^{*}\right) \quad \text { with }\left\|y^{*}\right\|=1 .
$$

This contradicts condition (b). Therefore, $(b) \Rightarrow(c)$.

Implications $(\mathrm{c}) \Rightarrow(\mathrm{d}) \Rightarrow(\mathrm{e})$ are obvious.

Let us prove (e) $\Rightarrow$ (a). Note, first of all, that (e) implies (b). Indeed, if $a(\Phi, \bar{x})=0$, then there exist (due to the boundedness of $\Phi(\bar{x})$ and the robustness of the coderivative) vectors $\bar{y} \in \Phi(\bar{x})$ and $y^{*} \in \mathbf{R}^{m}$ such that (3.8) holds true. The latter means that $\operatorname{Ker} D^{*} \Phi(\bar{x}, \bar{y}) \neq\{0\}$.

Now assuming (e), we prove that any positive number $a<a(\Phi, \bar{x})$ is a covering modulus for $\Phi$ in some neighborhood $U$ of $\bar{x}$.

Suppose that it is not true for some given positive number $a<a(\Phi, \bar{x})$. Then there exist sequences of the corresponding vectors $\left\{x_{k}\right\},\left\{y_{k}\right\},\left\{z_{k}\right\}$ and positive numbers $\left\{r_{k}\right\}$ such that $x_{k} \rightarrow \bar{x}, r_{k} \downarrow 0$, and conditions (3.7) are fulfilled. For each $k=1,2, \ldots$, let us consider the distance function

$$
\rho_{k}(x):=\operatorname{dist}\left(z_{k}, \Phi(x)\right) .
$$

Using this function, we can rewrite conditions (3.7) in the form

$$
\rho_{k}\left(x_{k}\right) \leq a r_{k}, \quad \rho_{k}(x)>0 \quad \text { for all } x \in B_{r_{k}}\left(x_{k}\right) \text { and } k=1,2, \ldots .
$$

Now let us apply Proposition 2.18 to the function

$$
\varphi(x):=\rho_{k}(x)+\delta\left(x, B_{r_{k}}\left(x_{k}\right)\right)
$$

where $\delta(\cdot, \cdot)$ is the indicator function of the given set. Putting

$$
\varepsilon=a r_{k}, \quad x_{\varepsilon}=x_{k}, \quad \lambda=r_{k}-r_{k}^{2}, \quad \text { and } \quad p(x)=\|x\|
$$

in Proposition 2.18, we find a vector $v_{k} \in \mathbf{R}^{n}$ such that

$$
\left\|v_{k}-x_{k}\right\| \leq r_{k}-r_{k}^{2}
$$

and $v_{k}$ is a solution of the following unconstrained minimization problem:

$$
\rho_{k}(x)+\delta\left(x, B_{r_{k}}\left(x_{k}\right)\right)+\frac{a}{1-r_{k}}\left\|x-v_{k}\right\| \rightarrow \inf , \quad x \in \mathbf{R}^{n} .
$$


Moreover, $v_{k} \in$ int $B_{r_{k}}\left(x_{k}\right)$, i.e., one can actually consider the function in (3.10) without the second term.

Using Proposition 2.8 in problem (3.10) and then Corollary 2.14, we obtain the relation

$$
\left\|x_{k}^{*}\right\| \leq a /\left(1-r_{k}\right) \text { for all } x_{k}^{*} \in \partial^{-} \rho_{k}\left(v_{k}\right) \text { and } k=1,2, \ldots .
$$

For evaluating the subdifferential of function (3.9) at the point $v_{k}$ under the condition $z_{k} \notin \Phi\left(v_{k}\right)$, we employ Corollary 2.17. By virtue of this result, there exist vectors $w_{k} \in P\left(z_{k}, \Phi\left(v_{k}\right)\right)$ and $y_{k}^{*} \in \mathbf{R}^{m}$ such that

$$
x_{k}^{*} \in D^{*} \Phi\left(v_{k}, w_{k}\right)\left(y_{k}^{*}\right) \quad \text { with }\left\|y_{k}^{*}\right\|=1 \text { for } k=1,2, \ldots .
$$

It follows from the construction above that $v_{k} \rightarrow \bar{x}$ as $k \rightarrow \infty$ and the sequences $\left\{w_{k}\right\},\left\{x_{k}^{*}\right\}$, and $\left\{y_{k}^{*}\right\}$ are bounded. Denote by $\bar{y}, x^{*}$, and $y^{*}$ their corresponding limiting points. It is clear that

$$
\left\|y^{*}\right\|=1, \quad \bar{y} \in \Phi(\bar{x}), \quad \text { and } \quad x^{*} \in D^{*} \Phi(\bar{x}, \bar{y})\left(y^{*}\right)
$$

(due to Proposition 2.6). From (3.11) and (3.1) one has

$$
a \geq\left\|x^{*}\right\| \geq a(\Phi, \bar{x})
$$

which ends the proof of implication (e) $\Rightarrow$ (a) .

The equality $(\operatorname{cov} \Phi)=a(\Phi, \bar{x})$ follows immediately from the proofs of implications $(a) \Rightarrow(b)$ and (e) $\Rightarrow(a)$ above. The second equality in (3.5) is induced by Proposition 3.1.

For the case of single-valued mappings we get the following result.

Corollary 3.4. Let $\Phi$ be single-valued and locally Lipschitzian around $\bar{x}$. Then for $\Phi$ enjoying the covering property, it is necessary and sufficient that each of the following equivalent conditions is fulfilled:

(a) One has

$$
a(\Phi, \bar{x})=\min \left\{\left\|x^{*}\right\|: x^{*} \in \partial^{-}\left\langle y^{*}, \Phi\right\rangle(\bar{x}),\left\|y^{*}\right\|=1\right\}>0 .
$$

(b) There exist a number $c>0$ and a neighborhood $U$ of $\bar{x}$ such that

$$
\left\|y^{*}\right\| \leq c\left\|x^{*}\right\| \quad \text { if } x^{*} \in \partial^{-}\left\langle y^{*}, \Phi\right\rangle(x) \text { and } x \in U \text {. }
$$

(c) There exists a neighborhood $U$ of $\bar{x}$ such that

$$
\left[0 \in \partial^{-}\left\langle y^{*}, \Phi\right\rangle(x) \text { and } x \in U\right] \Rightarrow y^{*}=0 .
$$

(d) One has

$$
\left[0 \in \partial^{-}\left\langle y^{*}, \Phi\right\rangle(\bar{x})\right] \Rightarrow y^{*}=0 .
$$

When these properties hold, then

$$
(\operatorname{cov} \Phi)(\bar{x})=a(\Phi, \bar{x})=1 / \min \left\{c:\left\|y^{*}\right\| \leq c\left\|x^{*}\right\| \text { if } x^{*} \in \partial^{-}\left\langle y^{*}, \Phi\right\rangle(\bar{x})\right\} .
$$

Proof. It follows from Theorem 3.3 by virtue of Proposition 2.12 .

In the smooth case we obtain from here the classical Ljusternik-Graves theorem and provide the exact evaluation of the covering bound. 
Corollary 3.5. Let $\Phi: \mathbf{R}^{n} \rightarrow \mathbf{R}^{m}$ be strictly differentiable at $\bar{x}$. Then the classical surjectivity condition (1.2) is a criterion for $\Phi$ enjoying the covering property around $\bar{x}$. In this case one has

$$
\begin{aligned}
(\operatorname{cov} \Phi)(\bar{x}) & =\min \left\{\left\|(\nabla \Phi(\bar{x}))^{*} y^{*}\right\|:\left\|y^{*}\right\|=1\right\} \\
& =1 / \min \left\{c:\left\|y^{*}\right\| \leq c\left\|(\nabla \Phi(\bar{x}))^{*} y^{*}\right\| \text { for any } y^{*} \in \mathbf{R}^{m}\right\} .
\end{aligned}
$$

Moreover, if $m=n$ and the matrix $\nabla \Phi(\bar{x})$ is nonsingular, then

$$
(\operatorname{cov} \Phi)(\bar{x})=1 /\left\|(\nabla \Phi(\bar{x}))^{-1}\right\| .
$$

Proof. If $\Phi$ is strictly differentiable at $\bar{x}$, then, due to Proposition 2.4, the criterion (e) in Theorem 3.3 is reduced to

$$
(\nabla \Phi(\bar{x}))^{*} y^{*}=0 \text { for any } y^{*} \in \mathbf{R}^{m}
$$

which coincides with the surjectivity condition (1.2). The equalities in (3.12) follow from (3.5), (3.1), and (3.2) by virtue of Proposition 2.4. If $m=n$ and $\nabla \Phi(\bar{x})$ is invertible, then

$$
c(\Phi, \bar{x})=\left\|(\nabla \Phi(\bar{x}))^{-1}\right\|
$$

due to the definition of the norm for the linear operator $A:=(\nabla \Phi(\bar{x}))^{*}$ from $\mathbf{R}^{n}$ to $\mathbf{R}^{n}$ with regard to the well-known relations

$$
\left(A^{*}\right)^{-1}=\left(A^{-1}\right)^{*} \text { and }\left\|A^{*}\right\|=\|A\| .
$$

This implies (3.13).

Now we shall consider a local counterpart of the covering property for a multifunction $\Phi$ around the given point $(\bar{x}, \bar{y})$ from its graph.

Definition 3.6. A multifunction $\Phi$ is said to be open at a linear rate around $(\bar{x}, \bar{y}) \in \operatorname{gph} \Phi$ if there exists a number $a>0$, a neighborhood $U$ of $\bar{x}$, and a neighborhood $V$ of $\bar{y}$ such that

$$
B_{a r}(\Phi(x) \cap V) \subset \Phi\left(B_{r}(x)\right) \text { for any }(x, r) \text { with } B_{r}(x) \subset U \text {. }
$$

Each of such numbers $a$ is called the openness modulus for $\Phi$ around $(\bar{x}, \bar{y})$. The supremum of all openness moduli is called the openness bound for $\Phi$ around $(\bar{x}, \bar{y})$ and is denoted by $($ ope $\Phi)(\bar{x}, \bar{y})$.

Consider the numbers

$$
\begin{aligned}
& a(\Phi, \bar{x}, \bar{y}):=\inf \left\{\left\|x^{*}\right\|: x^{*} \in D^{*} \Phi(\bar{x}, \bar{y})\left(y^{*}\right),\left\|y^{*}\right\|=1\right\}, \\
& c(\Phi, \bar{x}, \bar{y}):=\inf \left\{c:\left\|y^{*}\right\| \leq c\left\|x^{*}\right\| \text { if } x^{*} \in D^{*} \Phi(\bar{x}, \bar{y})\left(y^{*}\right)\right\} .
\end{aligned}
$$

Similarly to Proposition 3.2, one can prove that if $a(\Phi, \bar{x}, \bar{y})>0$, then

$$
c(\Phi, \bar{x}, \bar{y})=1 / a(\Phi, \bar{x}, \bar{y}) .
$$

Now let us obtain an analogue of Theorem 3.3 for the openness property of $\Phi$ around $(\bar{x}, \bar{y})$. 
Theorem 3.7. The following conditions are equivalent:

(a) $\Phi$ is open at a linear rate around $(\bar{x}, \bar{y})$.

(b) $a(\Phi, \bar{x}, \bar{y})>0$.

(c) There exists a number $c>0$, a neighborhood $U$ of $\bar{x}$, and a neighborhood $V$ of $\bar{y}$ such that

(3.17) $\left\|y^{*}\right\| \leq c\left\|x^{*}\right\|$ for all $x^{*} \in D^{*} \Phi(x, y)\left(y^{*}\right), x \in U$, and $y \in \Phi(x) \cap V$.

(d) There exists neighborhoods $U$ of $\bar{x}$ and $V$ of $\bar{y}$ such that

$$
\operatorname{Ker} D^{*} \Phi(x, y)=\{0\} \quad \text { for all } x \in U \text { and } y \in \Phi(x) \cap V \text {. }
$$

(e) $\operatorname{Ker} D^{*} \Phi(\bar{x}, \bar{y})=\{0\}$.

When these properties hold, one has

$$
(\text { ope } \Phi)(\bar{x}, \bar{y})=a(\Phi, \bar{x}, \bar{y})=1 / c(\Phi, \bar{x}, \bar{y}) \text {. }
$$

Proof. If $a>0$ is an openness modulus for $\Phi$ around $(\bar{x}, \bar{y})$, then $a \leq$ $a(\Phi, \bar{x}, \bar{y})$; therefore, (a) $\Rightarrow(\mathrm{b})$. This is actually proved in the first part of Theorem 3.3 for $\Phi(\bar{x})=\{\bar{y}\}$. The same is true for the implication (b) $\Rightarrow$ (c). Implications $(\mathrm{c}) \Rightarrow(\mathrm{d}) \Rightarrow(\mathrm{e})$ are obvious.

It is also clear that $(e) \Rightarrow$ (b). So, for ending the proof of the theorem it suffices to show that if $a(\Phi, \bar{x}, \bar{y})>0$, then any positive number $a<a(\Phi, \bar{x}, \bar{y})$ is an openness modulus for $\Phi$ around $(\bar{x}, \bar{y})$. Supposing the opposite, one finds sequences $x_{k} \rightarrow \bar{x}, y_{k} \rightarrow \bar{y}, z_{k} \rightarrow \bar{y}$, and $r_{k} \downarrow 0$ as $k \rightarrow \infty$ such that (3.7) is fulfilled. Then we consider the distance function (3.9) and follow the proof of the last part of Theorem 3.3 where $w_{k} \rightarrow \bar{y}$ as $k \rightarrow \infty$.

Next we shall study a property of multifunctions which occupies an intermediate position between the covering and openness properties considered above.

Definition 3.8. We say that a multifunction $\Phi$ is compact-open at a linear rate around $\bar{x} \in \operatorname{Dom} \Phi$ if, for every compact set $V \subset \mathbf{R}^{m}$, there exist a neighborhood $U$ of $\bar{x}$ and a number $a>0$ such that (3.14) is fulfilled.

It is easy to see that the covering property for $\Phi$ around $\bar{x}$ always implies the property of compact openness for this multifunction around the same point. Moreover, if $\Phi$ is locally bounded around $\bar{x}$, then the both properties coincide. Let us now consider the interrelation between the properties of openness and compact openness for arbitrary (closed-graph) multifunctions.

Theorem 3.9. The following assertions are equivalent:

(a) $\Phi$ is compact-open at a linear rate around $\bar{x}$.

(b) $\Phi$ is open at a linear rate around $(\bar{x}, \bar{y})$ for every $\bar{y} \in \Phi(\bar{x})$.

Proof. First we prove $(\mathrm{a}) \Rightarrow(\mathrm{b})$. For any vector $\bar{y} \in \Phi(\bar{x})$, let us consider the closed ball $B_{\varepsilon}(\bar{y})$ with $\varepsilon>0$. If $\Phi$ is compact-open around $\bar{x}$, then for the compact set $V=B_{\varepsilon}(\bar{y})$ one can find a neighborhood $U$ of $\bar{x}$ and a number $a>0$ such that (3.14) holds. Therefore, this number $a$ is an openness modulus for $\Phi$ around $(\bar{x}, \bar{y})$ with the neighborhood $U$ of $\bar{x}$ and the neighborhood $\widetilde{V}:=\operatorname{int} B_{\varepsilon}(\bar{y})$ of $\bar{y}$.

Now let us prove the opposite implication (b) $\Rightarrow$ (a) using the compactness arguments similar to Rockafellar [54, Theorem 2.2]. Given compact set $\bar{V} \subset$ $\mathbf{R}^{m}$, we suppose first that $\Phi(\bar{x}) \cap \bar{V} \neq \varnothing$ and employ the openness property 
(b) for each $\bar{y} \in \Phi(\bar{x}) \cap \bar{V}$. In this way one gets parametric families of (open) neighborhoods $\left\{V_{\bar{y}}\right\}$ of the points $\bar{y} \in \Phi(\bar{x}) \cap \bar{V}$, corresponding neighborhoods $\left\{U_{\bar{y}}\right\}$ of $\bar{x}$, and moduli $\left\{a_{\bar{y}}>0\right\}$ such that inclusion (3.14) is fulfilled with

$$
a=a_{\bar{y}}, \quad V=V_{\bar{y}}, \quad \text { and } \quad U=U_{\bar{y}} \text { for every } \bar{y} \in \Phi(\bar{x}) \cap \bar{V} .
$$

Because $\Phi(\bar{x}) \cap \bar{V}$ is compact, we can select from $\left\{V_{\bar{y}}\right\}$ a finite covering $\left\{V_{i}\right\}$, $i \in I$, of the set $\Phi(\bar{x}) \cap \bar{V}$. Let

$$
W:=\bigcup_{i \in I} V_{i}, \quad U^{\prime}:=\bigcap_{i \in I} U_{i}, \quad \text { and } \quad \bar{a}:=\min _{i \in I} a_{i}>0 .
$$

It is clear that $W$ and $U^{\prime}$ are open sets, and the following holds:

$$
B_{a r r}(\Phi(x) \cap W) \subset \Phi\left(B_{r}(x)\right) \text { for any }(x, r) \text { with } B_{r}(x) \subset U^{\prime} .
$$

Consider the compact set $\bar{V} \backslash W$ for which $\Phi(\bar{x}) \cap(\bar{V} \backslash W)=\varnothing$. Because gph $\Phi$ is closed, for any $z \in \bar{V} \backslash W$ one can find neighborhoods $\widetilde{V}_{z}$ of $z$ and $\widetilde{U}_{z}$ of $\bar{x}$ such that

$$
\Phi(x) \cap \widetilde{V}_{z}=\varnothing \quad \text { if } x \in \widetilde{U}_{z} \text { and } z \in \bar{V} \backslash W .
$$

From the family of neighborhoods $\left\{\widetilde{V}_{z}\right\}$ as $z \in \bar{V} \backslash W$, we select a finite covering $\left\{\widetilde{V}_{j}\right\}, j \in J$, of the compact set $\bar{V} \backslash W$. Considering the corresponding neighborhoods $\left\{\tilde{U}_{j}\right\}, j \in J$, of the point $\bar{x}$, we define

$$
\widetilde{U}:=\bigcap_{j \in J} \widetilde{U}_{j} \text { and } \bar{U}:=U^{\prime} \cap \tilde{U} .
$$

Then one has

$$
\Phi(x) \cap \bar{V} \subset \Phi(x) \cap W \text { if } x \in \tilde{U} .
$$

From here and (3.20) we obtain inclusion (3.14) with $a=\bar{a}>0, U=\bar{U}$, and $V=\bar{V}$ in the case when $\Phi(\bar{x}) \cap \bar{V} \neq \varnothing$. If $\Phi(\bar{x}) \cap \bar{V}=\varnothing$, then we have $W=\varnothing$ in the arguments above and one gets the same inclusion (3.14) with $\bar{U}=\widetilde{U}$. Therefore, $\Phi$ is compact-open at a linear rate around $\bar{x}$, i.e., (b) $\Rightarrow(a)$.

Using this result and Theorem 3.7, one can obtain effective criteria for the compact openness property.

Corollary 3.10. The following conditions are equivalent:

(a) $\Phi$ is compact-open at a linear rate around $\bar{x}$.

(b) For every compact set $V \subset \mathbf{R}^{m}$, there exists a number $c>0$ and a neighborhood $U$ of $\bar{x}$ such that (3.17) is fulfilled.

(c) For every compact set $V \subset \mathbf{R}^{m}$, there exists a neighborhood $U$ of $\bar{x}$ such that (3.18) is fulfilled.

(d) $\operatorname{Ker} D^{*} \Phi(\bar{x}, \bar{y})=\{0\}$ for all $\bar{y} \in \Phi(\bar{x})$.

Proof. If $\Phi$ is compact-open around $\bar{x}$, then it has the openness property around any point $(\bar{x}, \bar{y})$ by virtue of Theorem 3.9. For every given compact set $\bar{V} \subset \mathbf{R}^{m}$, we can use condition (c) in Theorem 3.7 at any point $(\bar{x}, \bar{y}) \in \operatorname{gph} \Phi$ with $\bar{y} \in \bar{V}$. Then following the arguments in Theorem 3.9 directly, we prove condition (b) in the corollary. Implications $(b) \Rightarrow(c) \Rightarrow(d)$ are trivial.

If (d) holds, then $\Phi$ is open at a linear rate around any point $(\bar{x}, \bar{y}) \in \operatorname{gph} \Phi$, due to Theorem 3.7. This implies condition (a) in the corollary by virtue of Theorem 3.9. 
Note that for multifunctions $\Phi$ locally bounded around $\bar{x}$, all conditions (a)-(d) in Corollary 3.10 are equivalent to the condition $a(\Phi, \bar{x})>0$ in Theorem 3.3.

Remark 3.11. Some constants similar to (3.15) were first introduced by Ioffe [21] in the case of Lipschitz continuous functions. In fact, that paper contains the lower estimate of the openness bound

$$
(\text { ope } \Phi)(\bar{x}, \bar{y}) \geq a(\Phi, \bar{x}, \bar{y})
$$

for single-valued functions $\Phi$. For the case of multifunctions $\Phi$, a lower estimate equivalent to (3.21) has been obtained in [23, Corollary 2.3] by reducing to the single-valued setting.

The constant $a(\Phi, \bar{x})$ in (3.1) is introduced by Mordukhovich in [38, 39] where the equality

$$
(\operatorname{cov} \Phi)(\bar{x})=a(\Phi, \bar{x})
$$

has been actually proved (cf. [38, Theorem 8] and [39, Theorem 5.2]).

\section{Metric Regularity}

In this section we shall study various modifications of the metric regularity property for arbitrary multifunctions $\Phi$ operating from $\mathbf{R}^{n}$ into $\mathbf{R}^{m}$. Let us consider the inverse multifunction $\Phi^{-1}$ from $\mathbf{R}^{m}$ to $\mathbf{R}^{n}$ satisfying the relation

$$
x \in \Phi^{-1}(y) \Leftrightarrow y \in \Phi(x) \Leftrightarrow(x, y) \in \operatorname{gph} \Phi .
$$

Definition 4.1. We say that

(a) $\Phi$ is locally-metrically regular around $(\bar{x}, \bar{y}) \in \operatorname{gph} \Phi$ with modulus $c>0$ if there exists a neighborhood $U$ of $\bar{x}$, a neighborhood $V$ of $\bar{y}$, and a number $\gamma>0$ such that

$$
\operatorname{dist}\left(x, \Phi^{-1}(y)\right) \leq c \operatorname{dist}(y, \Phi(x))
$$

for any $x \in U$ and $y \in V$ satisfying

$$
\operatorname{dist}(y, \Phi(x)) \leq \gamma .
$$

The infimum of all regularity moduli $c$ is called the bound of local-metric regularity for $\Phi$ around $(\bar{x}, \bar{y})$ and is denoted by $(\operatorname{lreg} \Phi)(\bar{x}, \bar{y})$.

(b) $\Phi$ is compact-metrically regular around $\bar{x} \in \operatorname{Dom} \Phi$ (relative to the image) if, for every compact set $V \subset \mathbf{R}^{m}$, there exists a neighborhood $U$ of $\bar{x}$ and positive numbers $c$ and $\gamma$ such that (4.2) holds for any $x \in U$ and $y \in V$ satisfying (4.3).

(c) $\Phi$ is global-metrically regular around $\bar{x} \in \operatorname{Dom} \Phi$ (relative to the image) with modulus $c>0$ if there exists a neighborhood $U$ of $\bar{x}$ and a number $\gamma>0$ such that (4.2) holds for any $x \in U$ and $y \in \mathbf{R}^{m}$ satisfying (4.3). The infimum of all such moduli $c$ is called the bound of global-metric regularity for $\Phi$ around $\bar{x}$ relative to the image and is denoted by $(\operatorname{greg} \Phi)(\bar{x})$.

Let us establish interrelations between the metric regularity properties under consideration and the openness (covering) properties of multifunctions from $\S 3$. 
Theorem 4.2. Let $\Phi$ be a multifunction from $\mathbf{R}^{n}$ into $\mathbf{R}^{m}$ with $\bar{x} \in \operatorname{Dom} \Phi$ and $(\bar{x}, \bar{y}) \in \operatorname{gph} \Phi$. Then the following assertions hold:

(a) $\Phi$ is global-metrically regular around $\bar{x}$ relative to the image with modulus $c$ if and only if $\Phi$ enjoys the covering property around $\bar{x}$ with modulus $a=1 / c$.

(b) $\Phi$ is local-metrically regular around $(\bar{x}, \bar{y})$ with modulus $c$ if and only if $\Phi$ is open at a linear rate around $(\bar{x}, \bar{y})$ with modulus $a=1 / c$.

(c) $\Phi$ is compact-metrically regular around $\bar{x}$ relative to the image if and only if $\Phi$ is compact-open around the same point.

Proof. First we shall prove assertion (a). Let $\Phi$ be global-metrically regular around $\bar{x}$ with modulus $c>0$. Given $c$, we find positive numbers $\varepsilon$ and $\gamma$ such that (4.2) holds for all $x \in U:=$ int $B_{\varepsilon}(\bar{x})$ and $y \in \mathbf{R}^{m}$ satisfying (4.3). Consider the number $\delta:=\min \{\varepsilon, \gamma c\}$ and the neighborhood $\widetilde{U}:=\operatorname{int} B_{\delta}(\bar{x})$ of $\bar{x}$. Let

$$
z \in B_{r / c}(\Phi(x)) \text { with such }(x, r) \text { that } B_{r}(x) \subset \tilde{U} \text {. }
$$

Then $x \in$ int $B_{\varepsilon}(\bar{x})$ and $\operatorname{dist}(z, \Phi(x)) \leq r / c \leq \gamma$. Using the global-metric regularity property from Definition 4.1 (c) with $y=z$, one has

$$
\operatorname{dist}\left(x, \Phi^{-1}(z)\right) \leq c \operatorname{dist}(z, \Phi(x)) \leq r .
$$

Selecting $w \in \Phi^{-1}(z)$ with $\|x-w\|=\operatorname{dist}\left(x, \Phi^{-1}(z)\right)$, we get $w \in B_{r}(x)$ and $z \in \Phi(w) \subset \Phi\left(B_{r}(x)\right)$. This implies the covering property from Definition 3.1 with $a=1 / c$ and $U=\operatorname{int} B_{\delta}(\bar{x})$.

Now let us assume that $\Phi$ enjoys the covering property with modulus $a>0$ in the neighborhood $U:=\operatorname{int} B_{\varepsilon}(\bar{x}), \varepsilon>0$. Then we put

$$
\delta:=\varepsilon / 2, \quad \tilde{U}:=\operatorname{int} B_{\delta}(\bar{x}), \quad \tilde{\gamma}:=a \varepsilon / 2
$$

and prove the global-metric regularity property (4.2) for $\Phi$ in the neighborhood $\tilde{U}$ with modulus $c=1 / a$ and number $\gamma=\tilde{\gamma}$ in (4.3). Indeed, consider any vectors $x \in \tilde{U}$ and $y \in \mathbf{R}^{m}$ such that

$$
\gamma:=\operatorname{dist}(y, \Phi(x)) \leq \tilde{\gamma} .
$$

Putting $r:=\gamma / a$, one has $y \in B_{a r}(x)$ and $B_{r}(x) \subset U$. Using the covering property, we can find such a vector $w \in B_{r}(x)$ that $y=\Phi(w)$. Then

$$
\operatorname{dist}\left(x, \Phi^{-1}(y)\right) \leq\|x-w\| \leq r=(1 / a) \operatorname{dist}(y, \Phi(x)) .
$$

This means the global-metric regularity property for $\Phi$ around $\bar{x}$ with modulus $c=1 / a$.

The proof of assertion (b) is quite similar to (a).

Now we shall prove assertion (c). First let us assume that $\Phi$ is compactmetrically regular around $\bar{x}$. Then, taking $V:=B_{\varepsilon}(\bar{y})$ with arbitrary $\varepsilon>0$ in the definition of compact-metric regularity, one can easily see that $\Phi$ is localmetrically regular around $(\bar{x}, \bar{y})$ for any $\bar{y} \in \Phi(\bar{x})$. According to the previous assertion (b), $\Phi$ is open at a linear rate around any such $(\bar{x}, \bar{y})$. Now using Theorem 3.9, we can conclude that $\Phi$ is compact-open around $\bar{x}$.

Next let us prove the opposite implication in assertion (c). If $\Phi$ is compactopen around $\bar{x}$, then, due to Theorem 3.9 and assertion (b) in Theorem 4.2, this multifunction is local-metrically regular around any point $(\bar{x}, \bar{y}) \in \operatorname{gph} \Phi$. Consider an arbitrary compact set $\bar{V} \subset \mathbf{R}^{m}$ and suppose first that $\Phi(\bar{x}) \cap \bar{V} \neq \varnothing$. 
Then we employ the local-metric regularity property for $\Phi$ around any $(\bar{x}, \bar{y})$ with $\bar{y} \in \Phi(\bar{x}) \cap \bar{V}$.

Using this property, one can find parametric families of neighborhoods $\left\{V_{\bar{y}}\right\}$ of the points $\bar{y} \in \Phi(\bar{x}) \cap \bar{V}$ and $\left\{U_{\bar{y}}\right\}$ of $\bar{x}$ as well as families of positive numbers $\left\{c_{\bar{y}}\right\}$ and $\left\{\gamma_{\bar{y}}\right\}$ such that estimate (4.2) holds true with $c=c_{\bar{y}}$ when

$$
x \in U_{\bar{y}}, \quad y \in V_{\bar{y}}, \quad \operatorname{dist}(y, \Phi(x)) \leq \gamma_{\bar{y}}, \quad \text { and } \quad \bar{y} \in \Phi(\bar{x}) \cap \bar{V} .
$$

Similarly to the proof of Theorem 3.9, we select from $\left\{V_{\bar{y}}\right\}$ a finite covering $\left\{V_{i}\right\}, i \in I$, of the set $\Phi(\bar{x}) \cap \bar{V}$ and consider the corresponding families $\left\{U_{i}\right\}$, $\left\{c_{i}\right\}$, and $\left\{\gamma_{i}\right\}$ as $i \in I$. Denoting

$$
W:=\bigcup_{i \in I} V_{i}, \quad U^{\prime}:=\bigcap_{i \in I} U_{i}, \quad \bar{c}:=\max _{i \in I} c_{i}<\infty, \quad \text { and } \quad \gamma^{\prime}:=\min _{i \in I} \gamma_{i}>0,
$$

we get the estimate

$$
\begin{aligned}
& \operatorname{dist}\left(x, \Phi^{-1}(y)\right) \leq \bar{c} \operatorname{dist}(y, \Phi(x)) \\
& \quad \text { if } x \in U^{\prime}, y \in W, \text { and } \operatorname{dist}(y, \Phi(x)) \leq \gamma^{\prime} .
\end{aligned}
$$

Consider now any point $z \in \bar{V} \backslash W$. Taking into account that $\Phi(\bar{x}) \cap$ $(\bar{V} \backslash W)=\varnothing$ and $\operatorname{gph} \Phi$ is closed, we can find such neighborhoods $\widetilde{V}_{z}$ of $z$ and $\widetilde{U}_{z}$ of $\bar{x}$ that

$$
\tilde{\gamma}_{z}:=\inf _{y \in \widetilde{V}_{z}, x \in \widetilde{U}_{z}}\{\operatorname{dist}(y, \Phi(x))\}>0 \text { for all } z \in \bar{V} \backslash W .
$$

Let us select a finite covering $\left\{\widetilde{V}_{j}\right\}, j \in J$, of the set $\bar{V} \backslash W$ and consider the corresponding neighborhoods $\widetilde{U}_{j}$ of $\bar{x}$ and numbers $\tilde{\gamma}_{j}$ in (4.5) as $j \in J$. Putting

$$
\tilde{\gamma}:=\min _{j \in J} \tilde{\gamma}_{j}>0 \text { and } \tilde{U}:=\bigcap_{j \in J} \widetilde{U}_{j}
$$

one has

$$
\operatorname{dist}(y, \Phi(x)) \geq \tilde{\gamma} \quad \text { for any } y \in \bar{V} \backslash W \text { and } x \in \tilde{U} .
$$

Let us finally set

$$
\bar{U}:=U^{\prime} \cap \widetilde{U}, \quad \bar{\gamma}:=\min \left\{\gamma^{\prime}, \tilde{\gamma}\right\}
$$

and consider any $\gamma \in(0, \bar{\gamma})$. It follows from (4.6) that if $x \in \bar{U}, y \in \bar{V}$, and $\operatorname{dist}(y, \Phi(x)) \leq \gamma$, then $y \in W$. Therefore, for such $x$ and $y$ we have estimate (4.2) by virtue of (4.4). This implies the compact-metric regularity property in Definition $4.1($ b) with

$$
V=\bar{V}, \quad U=\bar{U}, \quad c=\bar{c}, \quad \text { and } \quad \gamma \in(0, \bar{\gamma})
$$

and ends the proof of the theorem in the case when $\Phi(\bar{x}) \cap \bar{V} \neq \varnothing$.

If $\Phi(\bar{x}) \cap \bar{V}=\varnothing$, then we can put $W=\varnothing$ in the arguments above and obtain the compact-metric regularity property in (4.2), (4.3) with

$$
V=\bar{V}, \quad U=\tilde{U}, \quad c=\bar{c}, \quad \text { and } \quad \gamma \in(0, \tilde{\gamma}) .
$$

From the theorem obtained and the openness (covering) criteria in $\S 3$ we can derive the complete characterizations of the metric regularity properties under consideration with the evaluation of the exact bounds in terms of constants (3.1), (3.2) and (3.15), (3.16). 
Corollary 4.3. For $\Phi$ to be local-metrically regular around $(\bar{x}, \bar{y})$ with some modulus $c>0$, it is necessary and sufficient that each of the equivalent conditions (b)-(e) in Theorem 3.7 be fulfilled as well as $c(\Phi, \bar{x}, \bar{y})<\infty$. In this case one has

$$
(\operatorname{lreg} \Phi)(\bar{x}, \bar{y})=c(\Phi, \bar{x}, \bar{y})=1 / a(\Phi, \bar{x}, \bar{y}) .
$$

Proof. It follows from Theorems 4.2(b) and 3.7.

Corollary 4.4. $\Phi$ is compact-metrically regular around $\bar{x}$ relative to the image if and only if $\Phi$ is local-metrically regular around $(\bar{x}, \bar{y})$ for every $\bar{y} \in \operatorname{gph} \Phi$. For the fulfilment of this property, it is necessary and sufficient that each of the equivalent conditions (b)-(d) in Corollary 3.10 hold.

Proof. It follows from Theorem 4.2(c) and Corollary 3.10.

Corollary 4.5. Let $\Phi$ be locally bounded around $\bar{x}$. Then for $\Phi$ being globalmetrically regular around $\bar{x}$ relative to the image with some modulus $c>0$, it is necessary and sufficient that each of the equivalent conditions $(\mathrm{b})-(\mathrm{e})$ in Theorem 3.3 be fulfilled as well as $c(\Phi, \bar{x})<\infty$. In this case one has

$$
(\operatorname{greg} \Phi)(\bar{x})=c(\Phi, \bar{x})=1 / a(\Phi, \bar{x}) .
$$

Proof. It follows from Theorems 4.2(a) and 3.3.

Let us obtain another form for the representation of the regularity bounds $c(\Phi, \bar{x})$ and $c(\Phi, \bar{x}, \bar{y})$ from (3.2) and (3.16) in terms of the inverse mapping (4.1). Recall that the norm of any positive homogeneous multifunction $F$ is defined by

$$
\|F\|:=\sup \{\|y\|: y \in F(x),\|x\| \leq 1\} .
$$

The following result extends equality (3.13) in Corollary 3.5 for a general case of nonsmooth operators with the multivalued inverse.

Proposition 4.6. For any $\Phi, \bar{x} \in \operatorname{Dom} \Phi$, and $(\bar{x}, \bar{y}) \in \operatorname{gph} \Phi$ one has

$$
\begin{gathered}
c(\Phi, \bar{x}, \bar{y})=\left\|D^{*} \Phi^{-1}(\bar{y}, \bar{x})\right\|, \\
c(\Phi, \bar{x})=\sup \left\{\left\|D^{*} \Phi^{-1}(\bar{y}, \bar{x})\right\|: \bar{y} \in \Phi(\bar{x})\right\} .
\end{gathered}
$$

Proof. Let us prove (4.8). It follows from (4.1) that

$$
\left(-x^{*}, y^{*}\right) \in N((\bar{x}, \bar{y}) \mid \operatorname{gph} \Phi) \Leftrightarrow\left(y^{*},-x^{*}\right) \in N((\bar{y}, \bar{x}) \mid \operatorname{gph} \Phi),
$$

i.e.,

$$
y^{*} \in D^{*} \Phi^{-1}(\bar{y}, \bar{x})\left(x^{*}\right) \Leftrightarrow-x^{*} \in D^{*} \Phi(\bar{x}, \bar{y})\left(-y^{*}\right) .
$$

Therefore, we have to prove that

$$
c(\Phi, \bar{x}, \bar{y})=\sup \left\{\left\|y^{*}\right\|: \exists x^{*} \in D^{*} \Phi(\bar{x}, \bar{y})\left(y^{*}\right) \text { with }\left\|x^{*}\right\| \leq 1\right\} .
$$

Let $c$ be any positive number such that

$$
\left[x^{*} \in D^{*} \Phi(\bar{x}, \bar{y})\left(y^{*}\right)\right] \Rightarrow\left\|y^{*}\right\| \leq c\left\|x^{*}\right\| .
$$

Considering $x^{*}$ with $\left\|x^{*}\right\| \leq 1$ in (4.12), one gets

$$
c \geq \sup \left\{\left\|y^{*}\right\|: \exists x^{*} \in D^{*} \Phi(\bar{x}, \bar{y})\left(y^{*}\right),\left\|x^{*}\right\| \leq 1\right\} .
$$


This implies the right-side inequality $(\geq)$ in (4.11).

For proving the left-side inequality in (4.11), let us consider any number $c$ satisfying the strict inequality in (4.13) and show that the given $c$ satisfies (4.12). If (4.12) is not fulfilled for this $c$, then there exist vectors $\bar{x}^{*}$ and $\bar{y}^{*}$ such that

$$
\bar{x}^{*} \in D^{*} \Phi(\bar{x}, \bar{y})\left(\bar{y}^{*}\right) \text { and }\left\|\bar{y}^{*}\right\|>c\left\|\bar{x}^{*}\right\| .
$$

One can always assume that $\bar{x}^{*} \neq 0$ because otherwise (4.11) is trivial. Setting $\tilde{x}^{*}:=\bar{x}^{*} /\left\|\bar{x}^{*}\right\|$ and $\tilde{y}^{*}:=\bar{y}^{*} /\left\|\bar{x}^{*}\right\|$, we get

$$
\tilde{x}^{*} \in D^{*} \Phi(\bar{x}, \bar{y})\left(\tilde{y}^{*}\right), \quad\left\|\tilde{x}^{*}\right\|=1, \quad \text { and }\left\|\tilde{y}^{*}\right\|>c
$$

which contradicts the choice of $c$. This ends the proof of formula (4.8). The second formula (4.9) follows from (4.8) and the equality

$$
c(\Phi, \bar{x})=\sup \{c(\Phi, \bar{x}, \bar{y}): \bar{y} \in \Phi(\bar{x})\}
$$

Next we consider two more metric regularity properties for $\Phi$ which are nonlocal with respect to the domain.

Definition 4.7. We say that

(a) $\Phi$ is compact-metrically regular around $\bar{y} \in \operatorname{Im} \Phi$ (relative to the domain) if, for every compact set $U \subset \mathbf{R}^{n}$, there exists a neighborhood $V$ of $\bar{y}$ and positive numbers $\_$and $\gamma$ such that (4.2) holds for any $x \in U$ and $y \in V$ satisfying (4.3).

(b) $\Phi$ is global-metrically regular around $\bar{y} \in \operatorname{Im} \Phi$ (relative to the domain) with modulus $c>0$ if there exists a neighborhood $V$ of $\bar{y}$ and a number $\gamma>0$ such that (4.2) holds for any $y \in V$ and $x \in \mathbf{R}^{n}$ satisfying (4.3). The infimum of all such moduli $c$ is called the bound of global-metric regularity for $\Phi$ around $\bar{y}$ relative to the domain and is denoted by $(\overline{\operatorname{greg}} \Phi)(\bar{y})$.

Let us give useful reformulations of the metric regularity properties for $\Phi$ in a neighborhood of $\bar{y}$.

Proposition 4.8. The following assertions hold:

(a) $\Phi$ is compact-metrically regular around $\bar{y} \in \operatorname{Im} \Phi$ relative to the domain if and only if, for every compact set $U \subset \mathbf{R}^{n}$, there exists a neighborhood $V$ of $\bar{y}$ and a positive number $c$ such that (4.2) is fulfilled for any $y \in V$ and $x \in U$ satisfying the relation

$$
\Phi(x) \cap V \neq \varnothing .
$$

(b) $\Phi$ is global-metrically regular around $\bar{y} \in \operatorname{Im} \Phi$ relative to the domain with modulus $c>0$ if and only if there exists a neighborhood $V$ of $\bar{y}$ such that (4.2) is fulfilled for any $y \in V$ and $x \in \mathbf{R}^{n}$ satisfying (4.13).

(c) $\Phi$ is local-metrically regular around $(\bar{x}, \bar{y}) \in \operatorname{gph} \Phi$ with modulus $c>0$ if and only if there exists neighborhoods $V$ of $\bar{y}$ and $U$ of $\bar{x}$ such that (4.2) is fulfilled for any $y \in V$ and $x \in U$ satisfying (4.14).

Proof. Let us prove assertion (a). If $\Phi$ is compact-metrically regular around $\bar{y}$, then, for any given compactum $\bar{U} \subset \mathbf{R}^{n}$, we have such a neighborhood $\bar{V}$ of $\bar{y}$ and positive numbers $c, \gamma$ that (4.2) holds under the conditions $x \in \bar{U}$, $y \in \bar{V}$, and (4.3). Let us consider $\widetilde{V}:=\operatorname{int} B_{r}(\bar{y})$ for arbitrary $r \in(0, \gamma / 2)$ and show that

$$
[\forall x \in \bar{U} \text { with } \Phi(x) \cap \tilde{V}] \Rightarrow \operatorname{dist}(y, \Phi(x)) \leq \gamma \quad \text { for all } y \in \tilde{V}
$$


Indeed, selecting any $z \in \Phi(x) \cap \widetilde{V}$, one has

$$
\operatorname{dist}(y, \Phi(x)) \leq\|y-z\| \leq 2 r \leq \gamma \quad \text { if } y \in \widetilde{V} .
$$

Setting $V:=\bar{V} \cap \tilde{V}$, we get (4.2) for any $y \in V$ and $x \in \bar{U}$ satisfying (4.14).

Let us prove the opposite implication in (a). For the given compactum $\bar{U} \in$ $\mathbf{R}^{n}$, we can find such $V=\operatorname{int} B_{\varepsilon}(\bar{y})$ as $\varepsilon>0$, that (4.2) holds with some $c>0$ for any $y \in V$ and $x \in \bar{U}$ satisfying (4.14). Let us consider another neighborhood $V^{\prime}:=$ int $B_{\varepsilon / 2}(\bar{y})$ and the number $\gamma:=\varepsilon / 2$. We want to show that

$$
\left[\forall(x, y) \text { with } y \in V^{\prime} \text { and } \operatorname{dist}(y, \Phi(x)) \leq \gamma\right] \Rightarrow \Phi(x) \cap \operatorname{int} B_{\varepsilon}(\bar{y}) \neq \varnothing .
$$

Indeed, for any such $y$ one can select $z \in \Phi(x)$ satisfying to $\|y-z\|=$ $\operatorname{dist}(y, \Phi(x))$. For this $z$ we have

$$
\|z-\bar{y}\| \leq\|z-y\|+\|y-\bar{y}\|<\gamma+\varepsilon / 2 \leq \varepsilon .
$$

Therefore, $z \in$ int $B_{\varepsilon}(\bar{y})$ and $\Phi(x) \cap$ int $B_{\varepsilon}(\bar{y}) \neq \varnothing$. This ends the proof of assertion (a). The proof of the other assertions (b) and (c) is quite similar.

Now we can obtain the exhausting criteria for the compact- and global-metric regularity of $\Phi$ around $\bar{y}$ relative to the domain.

Theorem 4.9. The following conditions are equivalent:

(a) $\Phi$ is compact-metrically regular around $\bar{y}$ relative to the domain.

(b) $\Phi$ is local-metrically regular around $(\bar{x}, \bar{y})$ for every $\bar{x} \in \Phi^{-1}(\bar{y})$.

(c) For every compact set $U \subset \mathbf{R}^{n}$, there exists a number $c>0$ and $a$ neighborhood $V$ of $\bar{y}$ such that (3.17) is fulfilled.

(d) For every compact set $U \subset \mathbf{R}^{n}$, there exists a neighborhood $V$ of $\bar{y}$ such that (3.18) is fulfilled.

(e) $\operatorname{Ker} D^{*} \Phi(\bar{x}, \bar{y})=\{0\}$ for all $\bar{x} \in \Phi^{-1}(\bar{y})$.

Proof. To prove $(\mathrm{a}) \Rightarrow(\mathrm{b})$, it suffices to consider $U:=B_{\varepsilon}(\bar{x})$ for any $\bar{x} \in$ $\Phi^{-1}(\bar{y})$ and $\varepsilon>0$ in the definition of compact-metric regularity relative to the domain. If (b) holds, then for every given compact set $\bar{U} \subset \mathbf{R}^{n}$, we can employ (due to Corollary 4.3) condition (c) in Theorem 3.7 at any point $(\bar{x}, \bar{y}) \in$ $\operatorname{gph} \Phi$ with $\bar{x} \in \bar{U}$. Now following the arguments in Theorem 3.7, we prove implication (b) $\Rightarrow$ (c) in the theorem under consideration. Implications (c) $\Rightarrow$ (d) $\Rightarrow(e)$ are obvious.

If (e) is fulfilled, then one has (b) by virtue of Corollary 4.3. To prove (b) $\Rightarrow(a)$, we have to repeat the arguments in assertion (c) of Theorem 4.2, replacing the set $\Phi(\bar{x}) \cap \bar{V}$ by the set $\Phi^{-1}(\bar{y}) \cap \bar{U}$ for every compactum $\bar{U} \subset$ $\mathbf{R}^{n}$.

Let us consider the numbers

$$
\begin{aligned}
& \bar{a}(\Phi, \bar{y}):=\inf \left\{\left\|x^{*}\right\|: x^{*} \in D^{*} \Phi(\bar{x}, \bar{y})\left(y^{*}\right),\left\|y^{*}\right\|=1, \bar{x} \in \Phi^{-1}(\bar{y})\right\} \\
& \bar{c}(\Phi, \bar{y}):=\inf \left\{c:\left\|y^{*}\right\| \leq c\left\|x^{*}\right\| \text { if } x^{*} \in D^{*} \Phi(\bar{x}, \bar{y})\left(y^{*}\right), \quad \bar{x} \in \Phi^{-1}(\bar{y})\right\} .
\end{aligned}
$$

Theorem 4.10. Let $\Phi^{-1}$ be locally bounded around $\bar{y}$. Then for $\Phi$ being globalmetrically regular around $\bar{y}$ relative to the domain with some modulus $c>0$, it is necessary and sufficient that each of the equivalent conditions (a)-(e) in 
Theorem 4.9 be fulfilled with $U=\mathbf{R}^{n}$. Moreover, each of these conditions is equivalent to

$$
\bar{c}(\Phi, \bar{y})<\infty
$$

In this case one has

$$
\begin{aligned}
(\overline{\operatorname{greg}} \Phi)(\bar{y}) & =\bar{c}(\Phi, \bar{y})=1 / \bar{a}(\Phi, \bar{y}) \\
& =\sup \left\{\left\|D^{*} \Phi^{-1}(\bar{y}, \bar{x})\right\|: \bar{x} \in \Phi^{-1}(\bar{y})\right\} .
\end{aligned}
$$

Proof. It is obvious that the global-metric regularity of $\Phi$ around $\bar{y}$ always implies the corresponding compact-metric regularity. If $\Phi^{-1}$ is locally bounded around $\bar{y}$, then the opposite is also true. Indeed, due to the local boundedness property, there exist a compact set $\widetilde{U} \subset \mathbf{R}^{n}$ and a neighborhood $\widetilde{V}$ of $\bar{y}$ such that $\Phi^{-1}(y) \subset \widetilde{U}$ for all $y \in \widetilde{V}$. By virtue of (4.1), this means that

$$
[(\Phi(x) \cap \tilde{V} \neq \varnothing)] \Rightarrow x \in \widetilde{U} .
$$

Using now the equivalence of the compact-metric regularity property from Proposition 4.8(a), one can find, for the given compactum $U=\widetilde{U}$, a number $c>0$ and a neighborhood $V$ of $\bar{y}$ such that (4.2) is fulfilled if $y \in V$ and $x \in \widetilde{U}$ satisfies to (4.14). Putting $V^{\prime}:=V \cap \widetilde{V}$ and taking into account (4.19), we obtain the global-metric regularity property for $\Phi$ around $\bar{y}$ with the modulus $c$ and the neighborhood $V^{\prime}$. It is easy to see that criteria (c) and (d) in Theorem 4.9 are fulfilled with $U=\mathbf{R}^{n}$ if $\Phi^{-1}$ is locally bounded around $\bar{y}$.

Let us show that if $\Phi^{-1}$ is locally bounded around $\bar{y}$, then criterion (e) in Theorem 4.9 is equivalent to (4.17). First suppose that (e) does not hold, i.e.,

$$
0 \in D^{*} \Phi(\bar{x}, \bar{y})\left(\bar{y}^{*}\right) \text { for some } \bar{x} \in \Phi^{-1}(\bar{y}) \text { and } \bar{y}^{*} \neq 0 .
$$

Hence there are no such $c>0$ that

$$
\left\|y^{*}\right\| \leq c\left\|x^{*}\right\| \text { for all } x^{*} \in D^{*} \Phi(\bar{x}, \bar{y})\left(y^{*}\right) \text { and } x^{*} \in \Phi^{-1}(\bar{y}),
$$

i.e., $\bar{c}(\Phi, \bar{y})=\infty$ in (4.16). This means that $(4.17) \Rightarrow(\mathrm{e})$.

Now let us suppose that $\bar{c}(\Phi, \bar{y})=\infty$, i.e., there are no such $c>0$ that (4.21) holds. Therefore, one can find sequences of positive numbers $c_{k} \rightarrow \infty$ and vectors $\bar{x}_{k}, x_{k}^{*}, y_{k}^{*}$ with

$$
\begin{aligned}
& \bar{x}_{k} \in \Phi^{-1}(\bar{y}), \quad x_{k}^{*} \in D^{*} \Phi\left(\bar{x}_{k}, \bar{y}\right)\left(y_{k}^{*}\right), \text { and }\left\|x_{k}^{*}\right\|<1 / c_{k}\left\|y_{k}^{*}\right\| \\
& \text { for } k=1,2, \ldots \text {. }
\end{aligned}
$$

Putting $\bar{y}_{k}^{*}:=y_{k}^{*} /\left\|y_{k}^{*}\right\|$ and $\bar{x}_{k}^{*}:=x_{k}^{*} /\left\|y_{k}^{*}\right\|$, one has

(4.22) $\left\|\bar{x}_{k}^{*}\right\|<1 / c_{k} \quad$ with $\bar{x}_{k}^{*} \in D^{*} \Phi\left(\bar{x}_{k}, \bar{y}_{k}\right)\left(\bar{y}_{k}^{*}\right), x_{k} \in \Phi^{-1}(\bar{y})$, and $\left\|\bar{y}_{k}^{*}\right\|=1$.

Passing to the limit in (4.22) as $k \rightarrow \infty$ along convergent subsequences of $\left\{\bar{x}_{k}\right\},\left\{\bar{x}_{k}^{*}\right\}$, and $\left\{\bar{y}_{k}^{*}\right\}$, we get (4.20). Therefore, (e) $\Rightarrow(4.17)$.

To conclude the proof of the theorem, we have to check the equalities in (4.18). The left-hand equality in (4.18) follows from (4.7) and relations

$$
\bar{c}(\boldsymbol{\Phi}, \bar{y})=\sup \left\{c(\Phi, \bar{x}, \bar{y}): \bar{x} \in \Phi^{-1}(\bar{y})\right\},
$$

$$
(\overline{\operatorname{greg}} \Phi)(\bar{y})=\sup \left\{(\operatorname{lreg} \Phi)(\bar{x}, \bar{y}): \bar{x} \in \Phi^{-1}(\bar{y})\right\}
$$


which can be proved in the same way as Proposition 4.6 (the latter relationship holds if $\Phi^{-1}(\bar{y})$ is compact). The equality $\bar{c}(\Phi, \bar{y})=1 / \bar{a}(\Phi, \bar{y})$ is proved quite similarly to Proposition 3.2. The remaining equality in (4.18) follows from (4.8) and (4.23).

Similarly to Corollaries 3.4 and 3.5 , one can obtain corresponding corollaries of Theorems 4.9 and 4.10 for the cases of single-valued and smooth operators.

Now let us consider some realizations of the results obtained in the case of multifunctions with closed convex graphs.

Theorem 4.11. Let $\Phi$ have the convex graph and let $\bar{y} \in \operatorname{Im} \Phi$. Then the following conditions are equivalent:

(a) There exists a vector $\bar{x} \in \Phi^{-1}(\bar{y})$ such that $\Phi$ is local-metrically regular around $(\bar{x}, \bar{y})$ with some modulus $c>0$.

(b) $\Phi$ is compact-metrically regular around $\bar{y}$ relative to the domain.

(c) For every compact set $U \subset \mathbf{R}^{n}$, there exist a number $c>0$ and a neighborhood $V$ of $\bar{y}$ such that

$$
\left\|y^{*}\right\| \leq c\left\|x^{*}\right\| \quad \text { if }\left\langle x^{*}, x\right\rangle-\left\langle y^{*}, y\right\rangle=\max \left\{\left\langle x^{*}, u\right\rangle-\left\langle y^{*}, v\right\rangle:(u, v) \in \operatorname{gph} \Phi\right\}
$$

with $x \in U$ and $y \in \Phi(x) \cap V$.

(d) For every compact set $U \in \mathbf{R}^{n}$, there exists a neighborhood $V$ of $\bar{y}$ such that the implication

$$
\left[\left\langle y^{*}, y\right\rangle=\min \left\{\left\langle y^{*}, v\right\rangle: v \in \operatorname{Im} \Phi\right\}\right] \Rightarrow y^{*}=0
$$

holds if $y \in \Phi(x) \cap V$ with $x \in U$.

(e) One has

$$
\left[\left\langle y^{*}, \bar{y}\right\rangle=\min \left\{\left\langle y^{*}, v\right\rangle: v \in \operatorname{Im} \Phi\right\}\right] \Rightarrow y^{*}=0 .
$$

(f) $\bar{y}$ is an interior point of the set $\operatorname{Im} \Phi$.

When these properties hold, then

$$
\begin{aligned}
(\operatorname{lreg} \Phi)(\bar{x}, \bar{y}) & =\sup _{\left\|x^{*}\right\| \leq 1}\left\{\left\|y^{*}\right\|:\left\langle x^{*}, x-\bar{x}\right\rangle \leq\left\langle y^{*}, y-\bar{y}\right\rangle \forall(x, y) \in \operatorname{gph} \Phi\right\} \\
& \left.=1 / \inf _{\left\|y^{*}\right\|=1}\left\{\left\|x^{*}\right\|:\left\langle x^{*}, x-\bar{x}\right\rangle \leq\left\langle y^{*}, y-\bar{y}\right\rangle \forall(x, y) \in \operatorname{gph} \Phi\right\}\right) .
\end{aligned}
$$

If, moreover, $\Phi^{-1}$ is locally bounded around $\bar{y}$, then each of the conditions (a)-(f) is a criterion of the global-metric regularity for $\Phi$ around $\bar{y}$ relative to the domain with some modulus $c>0$. In this case the global regularity bound $(\overline{\mathrm{greg}} \Phi)(\bar{y})$ is computed from (4.26) by formula (4.24).

Proof. Due to Proposition 2.4, conditions (c)-(e) in the theorem under consideration coincide with the corresponding conditions (c)-(e) in Theorem 4.9. Therefore, each of them is a criterion of the compact-metric regularity for a convex-graph multifunction $\Phi$ around $\bar{x}$ relative to the domain. Note that condition (e) in Theorem 4.11 does not depend on $\bar{x} \in \Phi^{-1}(\bar{y})$. By virtue of Corollary 4.3, this implies the equivalence of conditions (a) and (b) in Theorem 4.11 .

Now let us show that $(e) \Leftrightarrow(f)$. There is a well-known necessary and sufficient condition for $\bar{y}$ being a solution to the convex optimization problem in (4.25): $-y^{*} \in N(\bar{y} \mid \operatorname{Im} \Phi)$. Therefore, (4.25) means that $N(\bar{y} \mid \operatorname{Im} \Phi)=\{0\}$. 
The latter is a criterion for $\bar{y}$ being an interior point of the set $\operatorname{Im} \Phi$. This ends the proof of the equivalence of all conditions (a)-(f) in Theorem 4.11.

The formulas in $(4.26)$ for $(\operatorname{lreg} \Phi)(\bar{x}, \bar{y})$ follow from $(4.7),(4.8),(3.15)$, and Proposition 2.4. The last assertion in the theorem about the global-metric regularity of $\Phi$ relative to the domain follows from Theorem 4.10.

Note that implications $(\mathrm{f}) \Rightarrow$ (b) in Theorem 4.11 is the content of the celebrated Robinson-Ursescu theorem; see [47, 62] and [2, §3.3].

\section{LIPSCHITZIAN BEHAVIOR OF MULTIFUNCTIONS}

In this final section of the paper we shall study the following three Lipschitzian properties of a multifunction $\Phi$ from $\mathbf{R}^{n}$ into $\mathbf{R}^{m}$ with the closed graph.

Definition 5.1. We say that

(a) $\Phi$ is pseudo-Lipschitzian around $(\bar{x}, \bar{y}) \in \operatorname{gph} \Phi$ with modulus $l>0$ if there exists a neighborhood $U$ of $\bar{x}$ and a neighborhood $V$ of $\bar{y}$ such that

$$
\Phi\left(x^{\prime}\right) \cap V \subset \Phi(x)+l\left\|x^{\prime}-x\right\| B \text { for any } x, x^{\prime} \in U \text {. }
$$

The infimum of all such moduli $l$ is called the bound of pseudo-Lipschitzness for $\Phi$ around $(\bar{x}, \bar{y})$ and is denoted by $(\operatorname{plip} \Phi)(\bar{x}, \bar{y})$.

(b) $\Phi$ is sub-Lipschitzian around $\bar{x} \in \operatorname{Dom} \Phi$ if, for every compact set $V \subset \mathbf{R}^{m}$, there exists a neighborhood $U$ of $\bar{x}$ and a number $l>0$ such that (5.1) is fulfilled.

(c) $\Phi$ is locally Lipschitzian around $\bar{x} \in \operatorname{Dom} \Phi$ with modulus $l>0$ if there exists a neighborhood $U$ of $\bar{x}$ such that (5.1) is fulfilled when $V=\mathbf{R}^{m}$. The infimum of all such moduli $l$ is called the bound of local Lipschitzness for $\Phi$ around $\bar{x}$ and is denoted by $(\operatorname{lip} \Phi)(\bar{x})$.

Both the properties of pseudo-Lipschitzness, introduced by Aubin [1], and sub-Lipschitzness, introduced by Rockafellar [54], extend the classical locally Lipschitzian behavior to the case of unbounded multifunctions. The following assertions can be found in Rockafellar [54, Theorems 2.1 and 2.2].

Proposition 5.2. For any closed-graph multifunction $\Phi$ one has

(a) $\Phi$ is locally Lipschitzian around $\bar{x}$ with some modulus $l$ if and only if $\Phi$ is locally bounded and sub-Lipschitzian around this point.

(b) $\boldsymbol{\Phi}$ is sub-Lipschitzian around $\bar{x}$ if and only if $\boldsymbol{\Phi}$ is pseudo-Lipschitzian around $(\bar{x}, \bar{y})$ with some modulus $l$ for every point $\bar{y} \in \Phi(\bar{x})$.

Now let us prove interrelations between the Lipschitzian properties of $\Phi$ and the metric regularity properties of the inverse mapping (4.1).

Theorem 5.3. The following assertions hold:

(a) $\Phi$ is pseudo-Lipschitzian around $(\bar{x}, \bar{y}) \in \operatorname{gph} \Phi$ with modulus $l$ if and only if $\Phi^{-1}$ is local-metrically regular around $(\bar{y}, \bar{x}) \in \operatorname{gph} \Phi^{-1}$ with the same modulus $c=l$.

(b) $\Phi$ is sub-Lipschitzian around $\bar{x} \in \operatorname{Dom} \Phi$ if and only if $\Phi^{-1}$ is compactmetrically regular around $\bar{x} \in \operatorname{Im} \Phi^{-1}$ relative to the domain.

(c) $\Phi$ is locally Lipschitzian around $\bar{x} \in \operatorname{Dom} \Phi$ with modulus $l$ if and only if $\Phi^{-1}$ is global-metrically regular around $\bar{x} \in \operatorname{Im} \Phi^{-1}$ relative to the domain with the same modulus $l$. 
Proof. It is easy to see that the Lipschitzian condition (5.1) can be rewritten in the form

$$
\operatorname{dist}(y, \Phi(x)) \leq l\left\|x-x^{\prime}\right\| \quad \text { for any } x, x^{\prime} \in U \text { and } y \in \Phi\left(x^{\prime}\right) \cap V .
$$

Due to (4.1), we have from here

$$
x^{\prime} \in \Phi^{-1}(y) \cap U \text { and } \operatorname{dist}(y, \Phi(x)) \leq l \operatorname{dist}\left(x, \Phi^{-1}(y) \cap B_{r}(\bar{x})\right)
$$

for all $x \in U, y \in V$, and $B_{r}(\bar{x}) \subset U$. One can see that

$$
\operatorname{dist}\left(x, \Phi^{-1}(y) \cap B_{r}(\bar{x})\right)=\operatorname{dist}\left(x, \Phi^{-1}(y)\right)
$$

if $x \in$ int $B_{r}(\bar{x})$ and $\Phi^{-1}(y) \cap$ int $B_{r}(\bar{x}) \neq \varnothing$. Therefore,

$$
\operatorname{dist}(y, \Phi(x)) \leq l \operatorname{dist}\left(x, \Phi^{-1}(y)\right)
$$

for all $y \in V$ and $x \in \widetilde{U}:=$ int $B_{r}(\bar{x})$ with $\Phi^{-1}(x) \cap \tilde{U} \neq \varnothing$. According to Proposition 4.8, this proves that the Lipschitzian properties for $\Phi$ in assertions (a)-(c) of the theorem imply the corresponding metric regularity for the inverse mapping $\Phi^{-1}$.

On the other hand, if $\Phi^{-1}$ has one of the metric regularity properties (a)(c) in the theorem under consideration, then estimate (5.3) is fulfilled for any $x \in U$ and $y \in V$ with $\Phi^{-1}(y) \cap U \neq \varnothing$. This implies immediately the Lipschitzian behavior (5.2) for the multifunction $\Phi$.

It follows from the results obtained that the metric regularity criteria in $\S 4$ provide necessary and sufficient conditions for the Lipschitzian behavior of the inverse mappings. Let us, in particular, formulate an effective criterion for the existence of the pseudo-Lipschitzian inverse (inverse mapping principle).

Corollary 5.4. For an arbitrary (closed-graph) multifunction $\Phi$ having the pseudoLipschitzian inverse $\Phi^{-1}$ around $(\bar{y}, \bar{x})$, it is necessary and sufficient that

$\operatorname{Ker} D^{*} \Phi(\bar{x}, \bar{y})=\{0\}$.

Proof. It follows from Theorem 5.3(a) and criterion (e) in Theorem 3.7 due to Corollary 4.3 .

Remark 5.5. If the coderivative $D^{*} \Phi$ is replaced in (5.4) by the Clarke coderivative (2.5), then one has the condition

$$
\operatorname{Ker} D_{C}^{*} \Phi(\bar{x}, \bar{y})=\{0\}
$$

which is sufficient for the existence of the pseudo-Lipschitzian inverse but is far removed from the necessity (see $\S 1$ ). In particular, condition (5.5) is never fulfilled in the case when the inverse function $\Phi^{-1}$ is single-valued and (nonsmooth) Lipschitz continuous around $\bar{y}$. Due to the duality between Clarke's normal and tangent cones, condition (5.5) is equivalent to the surjectivity condition

$$
\operatorname{Im} D_{C} \Phi(\bar{x}, \bar{y})=\mathbf{R}^{m}
$$

stated in terms of the Clarke (circatangent) derivative of the multifunction $\Phi$ (see, e.g., [3, Chapter 5]). Under this surjectivity condition, the existence of the pseudo-Lipschitzian inverse was first proved in Aubin [1]. 
Remark 5.6. If $\Phi: \mathbf{R}^{n} \rightarrow \mathbf{R}^{m}$ is single-valued and locally Lipschitzian around $\bar{x}$, then by virtue of Proposition 2.12, criterion (5.4) for $\Phi^{-1}$ being pseudoLipschitzian around $\bar{y}=\Phi(\bar{x})$ is reduced to

$$
\left[0 \in \partial^{-}\left\langle y^{*}, \Phi\right\rangle(\bar{x})\right] \Rightarrow y^{*}=0 .
$$

Due to formula (2.13), this criterion is automatically fulfilled under the rank condition (1.3). If $\Phi: \mathbf{R}^{n} \rightarrow \mathbf{R}^{n}$, then condition (1.3) means that any matrix $A \in J_{C} \Phi(\bar{x})$ is nonsingular. The latter implies, moreover, that $\Phi^{-1}$ is single-valued around $\Phi(x)$ (see Clarke [8, Theorem 7.1.1]). Note that Kummer [33] has recently obtained a necessary and sufficient condition for a locally Lipschitzian vector function $\Phi$ having the single-valued and Lipschitz continuous inverse. This condition is expressed in terms of the generalized directional derivative of $\Phi$ first introduced by Thibault [59].

Now using Theorem 5.3 and the corresponding criteria for the metric regularity of the inverse mappings, we obtain complete characterizations of the Lipschitzian properties for closed-graph multifunctions.

Theorem 5.7. The following conditions are equivalent:

(a) $\Phi$ is pseudo-Lipschitzian around $(\bar{x}, \bar{y})$ with some modulus $l>0$.

(b) One has

$$
c\left(\Phi^{-1}, \bar{y}, \bar{x}\right)=\inf \left\{c:\left\|x^{*}\right\| \leq c\left\|y^{*}\right\| \text { if } x^{*} \in D^{*} \Phi(\bar{x}, \bar{y})\left(y^{*}\right)\right\}<\infty .
$$

(c) There exist a neighborhood $U$ of $\bar{x}$, a neighborhood $V$ of $\bar{y}$, and a positive number $l$ such that

$$
\sup \left\{\left\|x^{*}\right\|: x^{*} \in D^{*} \Phi(x, y)\left(y^{*}\right)\right\} \leq l\left\|y^{*}\right\|
$$

for all $x \in U, y \in \Phi(x) \cap V$, and $y^{*} \in \mathbf{R}^{m}$.

(d) There exists neighborhoods $U$ of $\bar{x}$ and $V$ of $\bar{y}$ such that

$$
D^{*} \Phi(x, y)(0)=\{0\} \quad \text { for all } x \in U \text { and } y \in \Phi(x) \cap V .
$$

(e) $D^{*} \Phi(\bar{x}, \bar{y})(0)=\{0\}$.

If these properties hold, then

$$
(\operatorname{plip} \Phi)(\bar{x}, \bar{y})=\left\|D^{*} \Phi(\bar{x}, \bar{y})\right\|=c\left(\Phi^{-1}, \bar{y}, \bar{x}\right)=1 / a\left(\Phi^{-1}, \bar{y}, \bar{x}\right)
$$

where

$$
a\left(\Phi^{-1}, \bar{y}, \bar{x}\right)=\inf \left\{\left\|y^{*}\right\|: \exists x^{*} \in D^{*} \Phi(\bar{x}, \bar{y})\left(y^{*}\right) \text { with }\left\|x^{*}\right\|=1\right\}>0 .
$$

Proof. Criteria (b)-(e) for $\Phi$ being pseudo-Lipschitzian around $(\bar{x}, \bar{y})$ follow from Theorem 5.3(a) and Corollary 4.3 by virtue of formula (4.10). Due to Theorem 5.3(a), one has

$$
(\operatorname{plip} \Phi)(\bar{x}, \bar{y})=\left(\operatorname{lreg} \Phi^{-1}\right)(\bar{y}, \bar{x}) .
$$

Therefore, the equalities in (5.8) follow from (4.7), (4.8), and (4.10).

Remark 5.8. Estimate (5.6), first obtained in [37] for locally Lipschitzian multifunctions $\Phi$, means that the coderivative $D^{*} \Phi$ has a uniform boundedness property around $(\bar{x}, \bar{y})$. This is very important, in particular, for limiting processes involving adjoint variables (e.g., in problems of dynamic optimization and control). One can find some utilizations of this property in Mordukhovich $[39,41]$ for obtaining necessary optimality and controllability conditions in differential inclusions. Note that an analogue of (5.6) with the Clarke coderivative does not hold. 
Theorem 5.9. The following conditions are equivalent:

(a) $\Phi$ is sub-Lipschitzian around $\bar{x}$.

(b) For every compact set $V \subset \mathbf{R}^{m}$, there exist a neighborhood $U$ of $\bar{x}$ and a positive number $l$ such that estimate (5.6) is fulfilled.

(c) For every compact set $V \subset \mathbf{R}^{m}$, there exists a neighborhood $U$ of $\bar{x}$ such that (5.7) is fulfilled.

(d) $D^{*} \Phi(\bar{x}, \bar{y})(0)=\{0\}$ for all $\bar{y} \in \Phi(\bar{x})$.

Proof. It follows from Theorem 5.3(b) and Theorem 4.9 due to formula (4.10). These results may also be obtained from the criteria in Theorem 5.7 by using Proposition 5.3(b) (similarly to the arguments in Corollary 3.10).

Using this theorem, now we can provide an effective sufficient condition for the fulfillment of the main assumption (2.14) in Theorem 2.13 on the calculus of coderivatives.

Corollary 5.10. For any closed-graph multifunctions $\Phi_{1}$ and $\Phi_{2}$, property (2.14) holds if either $\Phi_{1}$ or $\Phi_{2}$ is sub-Lipschitzian around $\bar{x}$.

Proof. It follows from criterion (d) in Theorem 5.9.

Theorem 5.11. Let $\Phi$ be locally bounded around $\bar{x}$. Then the following conditions are equivalent:

(a) $\Phi$ is locally Lipschitzian around $\bar{x}$ with some modulus $l>0$.

(b) One has

$\bar{c}\left(\Phi^{-1}, \bar{x}\right)=\inf \left\{c:\left\|x^{*}\right\| \leq c\left\|y^{*}\right\|\right.$ if $\left.x^{*} \in D^{*} \Phi(\bar{x}, \bar{y})\left(y^{*}\right), \bar{y} \in \Phi(\bar{x})\right\}<\infty$.

(c) There exist a neighborhood $U$ of $\bar{x}$ and a positive number $l$ such that estimate (5.6) is fulfilled with $V=\mathbf{R}^{m}$. $\mathbf{R}^{m}$.

(d) There exists a neighborhood $U$ of $\bar{x}$ such that (5.7) is fulfilled with $V=$

(e) $D^{*} \Phi(\bar{x}, \bar{y})(0)=\{0\}$ for all $\bar{y} \in \Phi(\bar{x})$.

If these properties hold, then

$$
(\operatorname{lip} \Phi)=\sup \left\{\left\|D^{*} \Phi(\bar{x}, \bar{y})\right\|: \bar{y} \in \Phi(\bar{x})\right\}=\bar{c}\left(\Phi^{-1}, \bar{x}\right)=1 / \bar{a}\left(\Phi^{-1}, \bar{x}\right)
$$

where

$$
\bar{a}\left(\Phi^{-1}, \bar{x}\right)=\inf \left\{\left\|y^{*}\right\|: \exists x^{*} \in D^{*} \Phi(\bar{x}, \bar{y})\left(y^{*}\right) \text { with }\left\|x^{*}\right\| \leq 1, \bar{y} \in \Phi(\bar{x})\right\}>0 .
$$

Proof. Criteria (b)-(e) for $\Phi$ being locally Lipschitzian around $\bar{x}$ follow from Theorem 5.3(c) and Theorem 4.10 due to formula (4.10). They may also be derived directly from Theorem 5.9 by virtue of Proposition 5.2(a). Due to Theorem 5.3(c), we have

$$
(\operatorname{lip} \Phi)(\bar{x})=\left(\overline{\operatorname{greg}} \Phi^{-1}\right)(\bar{x}) .
$$

Therefore, the equalities in (5.9) follow from (4.18) and (4.10).

Now we formulate a corollary of the results obtained for multifunctions with closed convex graphs. 
Theorem 5.12. Let $\Phi$ have the convex graph and let $\bar{x} \in \operatorname{Dom} \Phi$. Then the following conditions are equivalent:

(a) There is a vector $\bar{y} \in \Phi(\bar{x})$ such that $\Phi$ is pseudo-Lipschitzian around $(\bar{x}, \bar{y})$ with some modulus $l>0$.

(b) $\Phi$ is sub-Lipschitzian around $\bar{x}$.

(c) For every compact set $V \subset \mathbf{R}^{m}$, there exists a positive number $l$ and a neighborhood $U$ of $\bar{x}$ such that

$$
\left\|x^{*}\right\| \leq\left\|y^{*}\right\| \quad \text { if }\left\langle x^{*}, x\right\rangle-\left\langle y^{*}, y\right\rangle=\max \left\{\left\langle x^{*}, u\right\rangle-\left\langle y^{*}, v\right\rangle:(u, v) \in \operatorname{gph} \Phi\right\}
$$

with $x \in U$ and $y \in \Phi(x) \cap V$.

(d) For every compact set $V \in \mathbf{R}^{m}$, there exists a neighborhood $U$ of $\bar{x}$ such that the implication

$$
\left[\left\langle x^{*}, x\right\rangle=\max \left\{\left\langle x^{*}, u\right\rangle: u \in \operatorname{Im} \Phi^{-1}\right\}\right] \Rightarrow x^{*}=0
$$

holds if $x \in \Phi^{-1}(y) \cap U$ with $y \in V$.

(e) One has

$$
\left[\left\langle x^{*}, \bar{x}\right\rangle=\max \left\{\left\langle x^{*}, u\right\rangle: u \in \operatorname{Im} \Phi^{-1}\right\}\right] \Rightarrow x^{*}=0 .
$$

(f) $\bar{x}$ is an interior point of the set $\operatorname{Im} \Phi^{-1}$.

When these properties hold, then

(5.10)

$$
\begin{aligned}
(\operatorname{plip} \Phi)(\bar{x}, \bar{y}) & =\sup _{\left\|y^{*}\right\| \leq 1}\left\{\left\|x^{*}\right\|:\left\langle x^{*}, x-\bar{x}\right\rangle \leq\left\langle y^{*}, y-\bar{y}\right\rangle \forall(x, y) \in \operatorname{gph} \Phi\right\} \\
& =1 / \inf _{\left\|x^{*}\right\|=1}\left\{\left\langle x^{*}, x-\bar{x}\right\rangle \leq\left\langle y^{*}, y-\bar{y}\right\rangle \forall(x, y) \in \operatorname{gph} \Phi\right\} .
\end{aligned}
$$

If, moreover, $\Phi$ is locally bounded around $\bar{x}$, then each of the conditions (a)(f) above is a criterion for $\Phi$ being locally Lipschitzian around $\bar{x}$ with some modulus $l>0$. In this case the bound of local Lipschitzness for $\Phi$ around $\bar{x}$ is computed from (5.10) by the formula

$$
(\operatorname{lip} \Phi)(\bar{x})=\sup \{(\operatorname{plip} \Phi)(\bar{x}, \bar{y}): \bar{y} \in \Phi(\bar{x})\} .
$$

Proof. It follows directly from Theorems 4.11 and 5.3. It may also be obtained from Theorems 5.7, 5.9, and 5.11 due to Proposition 2.4.

Remark 5.13. Using the results obtained in $\S \S 3-5$ and the calculus rules for the coderivative and subdifferentials introduced ( $\$ 2)$, one can prove the corresponding criteria for the covering (openness), metric regularity, and Lipschitzian properties for multifunctions represented in the form of various compositions and combinations with other single-valued and multivalued mappings. In this way, in $[40,42]$ we study some problems of the sensitivity analysis for constraint systems depending on parameters which include, in particular, various perturbations in optimization problems and generalized equations.

Remark 5.14. If a multifunction $\Phi$ can be represented as the subdifferential (superdifferential) mapping

$$
\Phi(x):=\partial^{-} \varphi(x) \text { or } \Phi(x):=\partial^{+} \varphi(x)
$$

with some extended-real-valued function $\varphi$, then its coderivative $D^{*} \Phi$ is expressed in terms of the second-order semidifferentials (2.9) and (2.10). Therefore, we employ the second-order semidifferentials introduced for formulating 
the criteria in $\S \S 3-5$ for these "subdifferential-type" multifunctions. Note that such kinds of multifunctions occur in studying parametrized variational inequalities, gradient inclusions, complementarity problems, etc. (see examples and applications in [2, 27, 42, 49] and elsewhere).

\section{REFERENCES}

1. J.-P. Aubin, Lipschitz behavior of solutions to convex minimization problems, Math. Oper. Res. 9 (1984), 87-111.

2. J.-P. Aubin and I. Ekeland, Applied nonlinear analysis, Wiley, 1984.

3. J.-P. Aubin and H. Frankowska, Set-valued analysis, Birkhäuser, Boston, Mass., 1990.

4. J. M. Borwein, Stability and regular points of inequality systems, J. Optim. Theory Appl. 48 (1986), 9-52.

5. J. M. Borwein and D. M. Zhuang, Verifiable necessary and sufficient conditions for regularity of set-valued and single-valued maps, J. Math. Anal. Appl. 134 (1988), 441-459.

6. J. V. Burke, An exact penaiization viewpoint of constrained optimization, SIAM J. Control Optim. 29 (1991), 968-998.

7. F. H. Clarke, Generalized gradients and applications, Trans. Amer. Math. Soc. 205 (1975), 247-262.

8. __ Optimization and nonsmooth analysis, Wiley, 1983.

9. __, Methods of dynamic and nonsmooth optimization, Soc. Indust. Appl. Math., Philadelphia, Pa., 1989.

10. R. Cominetti, Metric regularity, tangent sets, and second-order optimality conditions, Appl. Math. Optim. 21 (1990), 265-287.

11. A. V. Dmitruk, A. A. Miljutin, and N. P. Osmolovskii, Ljusternik's theorem and the theory of extrema, Russian Math. Surveys 35 (1980), 11-51.

12. A. L. Dontchev and W. W. Hager, Lipschitzian stability in nonlinear control and optimization, SIAM J. Control Optim. 31 (1993), 569-603.

13. I. Ekeland, On the variational principle, J. Math. Anal. Appl. 47 (1974), 324-358.

14. A. V. Fiacco, Introduction to sensitivity analysis in mathematical programming, Academic Press, 1983.

15. H. Frankowska, An open mapping principle for set-valued maps, J. Math. Anal. Appl. 127 (1987), 172-180.

16. , Some inverse mapping theorems, Ann. Inst. H. Poincaré Anal. Non Linéaire 7 (1990), 183-234.

17. L. M. Graves, Some mapping theorems, Duke Math. J. 17 (1950), 111-114.

18. H. Halkin, Interior mapping theorem with set-valued derivatives, J. Analyse Math. 30 (1975), 200-207.

19. J.-B. Hiriart-Urruty, $A$ short proof of the variational principle for approximate solutions of a minimization problem, Amer. Math. Monthly 90 (1983), 206-207.

20. A. D. Ioffe, Regular points of Lipschitz functions, Trans. Amer. Math. Soc. 251 (1979), 61-69.

21. __ Nonsmooth analysis: differential calculus of nondifferentiable mappings, Trans. Amer. Math. Soc. 266 (1981), 1-56.

22. __ Approximate subdifferentials and applications. I: The finite dimensional theory, Trans. Amer. Math. Soc. 281 (1984), 389-416.

23. __ On the local surjection property, Nonlinear Anal. 11 (1987), 565-592.

24. __, Approximate subdifferentials and applications. III: The metric theory, Mathematika 36 (1989), 1-38.

25. A. D. Ioffe and V. M. Tihomirov, Theory of extremal problems, North-Holland, Amsterdam, 1979. 
26. A. Jourani and L. Thibault, Approximate subdifferentials and metric regularity: the finite dimensional case, Math. Programming 47 (1990), 203-218.

27. A. J. King and R. T. Rockafellar, Sensitivity analysis for nonsmooth generalized equations, Math. Programming 55 (1992), 193-212.

28. A. Kruger, Properties of generalized differentials, Siberian Math. J. 26 (1985), 822-832.

29. _ A covering theorem for set-valued mappings, Optimization 19 (1988), 763-780.

30. A. Kruger and B. Mordukhovich, Generalized normals and derivatives, and necessary conditions for extrema in nondifferentiable programming. I, Depon. VINITI No. 408-80, Moscow, 1980. (Russian)

31. Generalizes normals and derivatives, and necessary conditions for extrema in nondifferentiable programming. II, Depon. VINITI No. 494-80, Moscow, 1980. (Russian)

32. _ Extremal points and the Euler equation in nonsmooth optimization, Dokl. Akad. Nauk BSSR 24 (1980), 684-687. (Russian)

33. B. Kummer, An implicit function theorem for $C^{0,1}$-equations and parametric $C^{1,1}$-optimization, J. Math. Anal. Appl. 158 (1991), 35-46.

34. L. A. Ljusternik, Conditional extrema of functionals, Math. USSR-Sb. 41 (1934), 390-401.

35. G. J. Minty, Monotone (nonlinear) operations in Hilbert space, Duke Math. J. 29 (1962), 341-346.

36. B. Mordukhovich, The maximum principle in the problem of time-optimal control with nonsmooth constraints, J. Appl. Math. Mech. 40 (1976), 960-969.

37. _ Metric approximations and necessary optimality conditions for general classes of nonsmooth extremal problems, Soviet Math. Dokl. 22 (1980), 526-530.

38. __ Nonsmooth analysis with nonconvex generalized differentials and adjoint mappings, Dokl. Akad. Nauk BSSR 28 (1984), 976-979. (Russian)

39. _ Approximation methods in problems of optimization and control, "Nauka", Moscow, 1988. (Russian; English translation to appear in Wiley-Interscience.)

40. __ Sensitivity analysis in nonsmooth optimization, Theoretical Aspects of Industrial Design (D. A. Field and V. Komkov, eds.), SIAM Proc. Appl. Math., vol. 58, SIAM, Philadelphia, Pa., 1992, pp. 32-46.

41. _ On variational analysis of differential inclusions, Optimization and Nonlinear Analysis (A. Ioffe, L. Marcus, and S. Reich, eds.), Pitman Res. Notes Math. Ser. 244, 1992, pp. 199-213.

42. _ Lipschitzian stability of constraint systems and generalized equations, Nonlinear Anal. (to appear).

43. __ Generalized differential calculus for nonsmooth and set-valued mappings, J. Math. Anal. Appl. (to appear).

44. J. J. Moreau, Fonctionelles convexes, Collége de France, Paris, 1966.

45. J.-P. Penot, Metric regularity, openness and Lipschitzian behavior of multifunctions, Nonlinear Anal. 13 (1989), 629-643.

46. B. H. Pourciau, Analysis and optimization of Lipschitz continuous mappings, J. Optim. Theory Appl. 22 (1977), 433-452.

47. S. M. Robinson, Regularity and stability of convex multivalued functions, Math. Oper. Res. 1 (1976), 130-145.

48. __ Stability theory for systems of inequalities. II: Differentiable nonlinear systems, SIAM J. Numer. Anal. 13 (1976), 497-513.

49. $\_$Generalized equations and their solutions. I: Basic theory, Math. Programming Stud. 10 (1979), 128-141.

50. R. T. Rockafellar, Convex analysis, Princeton Univ. Press, Princeton, N.J., 1970.

51. _ The theory of subgradients and its applications to problems of optimization: convex and nonconvex functions, Heldermann-Verlag, Berlin, 1981.

52. __ Proximal subgradients, marginal functions, and augmented Lagrangians in nonconvex optimization, Math. Oper. Res. 6 (1981), 427-437. 
53. $\ldots$ Extension of subgradient calculus with applications to optimization, Nonlinear Anal. 9 (1985), 665-698.

54. _ Lipschitzian properties of multifunctions, Nonlinear Anal. 9 (1985), 867-885.

55. Maximal monotone relations and the second derivatives of nonsmooth functions, Ann. Inst. H. Poincaré Anal. Non Linéaire 2 (1985), 167-184.

56. _ Proto-differentiability of set-valued mappings and its applications in optimization, Ann. Inst. H. Poincaré Anal. Non Linéaire 6 (1989), 449-482.

57. $\ldots$, Dualization of subgradient conditions for optimality, Nonlinear Anal. 20 (1993), 627-646.

58. A. Shapiro and J. F. Bonnans, Sensitivity analysis of parametrized programs under cone constraints, SIAM J. Control Optim. 30 (1992), 1409-1422.

59. L. Thibault, Subdifferentials of compactly Lipschitzian functions, Ann. Mat. Pura Appl. (4) 125 (1980), 157-192.

60. __ On subdifferentials of optimal value functions, SIAM J. Control Optim. 29 (1991), 1019-1036.

61. J. S. Treiman, Clarke's gradients and epsilon-subgradients in Banach spaces, Trans. Amer. Math. Soc. 294 (1986), 65-78.

62. C. Ursescu, Multifunctions with closed convex graph, Czechoslovak Math. J. 25 (1975), 438-441.

63. J. Warga, Fat homeomorphisms and unbounded derivate containers, J. Math. Anal. Appl. 81 (1981), 545-560.

Department of Mathematics, Wayne State University, Detroit, Michigan 48202

E-mail address: boris@math.wayne.edu 